\title{
Evaporation and condensation of spherical interstellar clouds. Self-consistent models with saturated heat conduction and cooling ${ }^{\star}$
}

\author{
W. Vieser ${ }^{1,2}$ and G. Hensler ${ }^{1}$ \\ ${ }^{1}$ Institute of Astronomy, University of Vienna, Türkenschanzstr. 17, 1180 Vienna, Austria \\ e-mail: hensler@astro.univie.ac.at \\ ${ }^{2}$ Christoph-Probst-Gymnasium, Talhofstr. 7, 82205 Gilching, Germany
}

Received 11 September 2005 / Accepted 17 August 2007

\begin{abstract}
Aims. The fate of interstellar clouds embedded in a hot tenuous medium depends on whether the clouds suffer from evaporation or whether material condensates onto the clouds. The knowledge of the evaporation or condensation rates of interstellar clouds at rest is therefore of prime importance for their further evolution. Analytic solutions for the rate of evaporative mass loss from an isolated spherical cloud embedded in a hot tenuous gas are deduced by Cowie \& McKee (1977, ApJ, 211, 135). Their approach is limited to the integration of the time-independent energy conservation equation for the heat-conductive interface. Therefore it is crucial to test the validity of the analytical results for more realistic interstellar conditions. This requires that the full hydrodynamical equations must be treated taking the whole cloud into account with a sufficiently large hot-gas reservoir.

Methods. By two-dimensional numerical simulations in an Eulerian, explicit hydrodynamical grid the evolution of interstellar clouds with different internal density structures and surrounded by a hot plasma is simulated. Self-gravity, interstellar heating and cooling effects and heat conduction by electrons are added. We use the classical thermal conductivity of a fully ionized hydrogen plasma proposed by Spitzer and a saturated heat flux according to Cowie and McKee in regions where the mean free path of the electrons is large compared to the temperature scaleheight.

Results. Using pure hydrodynamics and taking only the classical heat flux into account, we can reproduce the Cowie and McKee analytical results. If we allow for heat flux saturation the evaporation rate is reduced, but in the simulations even to about one order of magnitude below the predicted saturated one. This happens because the saturated heat flux is density dependent and due to the mixing of the two phases, the warm cloud material on one side and the hot intercloud medium, also the density distribution changes drastically there during the simulation. And this cannot be considered in the analytical study. This main result still holds if we add self gravity or choose another cloud density structure while keeping the cloud radius and temperature of the cloud edge constant. As a further issue the evolution changes, however, totally for more realistic conditions when interstellar heating and cooling effects stabilize the self-gravity. The clouds' evaporation then turns into condensation, because the additional energy input due to heat conduction can be transported away from the interface and radiated off very efficiently from the cloud's inner parts.

Conclusions. The assumption of pure classical heat conduction is invalid for the description of the evolution of interstellar clouds in a hot tenuous gas. The consideration of a limited saturated heat flux is inevitable for this kind of simulations and leads to a dramatic decrease of the evaporation rate. And even more realisticly with radiative cooling heat conduction leads to condensation in contradiction to analytical predictions which require evaporation. This has two consequences: Interstellar clouds are stabilized against evaporation. On the other hand this provides an efficient way to accrete and mix intercloud material into clouds.
\end{abstract}

Key words. ISM: clouds - ISM: structure - conduction - hydrodynamics - methods: numerical

\section{Introduction}

The Interstellar Medium (ISM) can be described as an inhomogeneous ensemble of three phases (McKee \& Ostriker 1977). The cold neutral phase with temperature $T \sim 80 \mathrm{~K}$ and density $n \sim 40 \mathrm{~cm}^{-3}$ is represented by the cores of molecular clouds which are confined by a warm neutral to slightly ionized medium $\left(T \sim 8000 \mathrm{~K}, n \sim 0.3 \mathrm{~cm}^{-3}\right)$. These two components are in pressure equilibrium if the gas is externally heated and can cool radiatively (Field et al. 1969). According to McKee and Ostriker they are embedded in a third phase of the ISM: the hot dilute intercloud medium (HIM) with $T \sim 10^{6} \mathrm{~K}$ and $n \sim$ $10^{-3}-10^{-4} \mathrm{~cm}^{-3}$. Since this component is produced locally by

^ Appendix is only available in electronic form at http://www . aanda.org supernova explosions at even higher temperatures and densities, it can originally not be in pressure equilibrium with the cooler phases and has therefore to expand vehemently. During this expansion shocks arise and the HIM penetrates through the ambient clumpy ISM. Denser clouds cannot be swept-up but are passed by the HIM so that they become embedded therein. Due to the strong discrepancy of the physical states between the phases an interface has to form where the hot phase and the molecular cloud are in contact. Temperatures and densities are connected through steep gradients that lead to energy and mass transfer. At this interfaces heat conduction plays an important role and is of prime importance whether the clouds evaporate or condense and therefore grow in mass. Analytical approximations require that small clouds in a hot plasma suffer from evaporation and will 
therefore be only short lived. The fact that interstellar clouds embedded in a hot medium are observed on the other hand suggests, that they are stabilized against evaporation so that their destruction is delayed.

These observations include High-Velocity Clouds (HVCs), HI entities consisting of a multi-phase structure (Wakker \& Schwarz 1991) and characterized by radial velocities that are incompatible with simple models of the differential rotation of the galactic disk (see Wakker \& van Woerden 1997 for a recent review). Distance measurements of the cloud complexes remain difficult. For at least two of them upper limits for their distances are deduced by Danly et al. (1993), Keenan et al. (1995) and van Woerden et al. (1997) which locate them in the hot galactic halo. Therefore, interactions with the hot rarefied halo gas are expected. Indications for such interactions are the detection of morphological perculiarities like head-tail structures (Brüns et al. 2000, 2001). Interactions with gas belonging to the galactic disk and having lower velocities are deduced from the observation of so-called velocity bridges (Pietz et al. 1996), fusions of high- and low-velocity gas in the velocity-position space.

Further examples for interstellar clouds in a stream of hot gas are cometary globules, like G134.6+1.4, which is located in a galactic chimney (Normandeau et al. 1996). This chimney is associated with the HII region W4 and forms a cone in the HI layer above W4 with a diameter of about $100 \mathrm{pc}$. At the bottom of the chimney the starcluster IC 1805 with 9 O stars can be found (Heyer et al. 1996; Taylor et al. 1999). An intense interaction between the shocked hot stellar winds and G134.6+1.4 is expected. Energy exchange between the cold molecular to neutral gas of the globule and the hot chimney gas leads either to an evaporation and therefore a destruction of the cloud what is not observed or to condensation of hot gas onto the globule and therefore to a stabilization.

Previous papers dealing with the evaporation and condensation of molecular clouds only solve the time-independent energy equation without taking temporal effects into account like mass changes, heating, etc., and dynamical effects like mixing of warm cloud material with the hot plasma and surface instabilities. In this paper we examine the evolution of interstellar clouds that are embedded in a hot, dilute medium by solving the full hydrodynamical equations numerically and furthermore following the heat conduction process in detail. In Sect. 2 we discuss the predicted evolution that is expected refering to the papers of Cowie \& McKee (1977, hereafter: CM77) and Dalton \& Balbus (1993, hereafter: DB93). The treatment of heat conduction in the context of hydrodynamical simulations is described in Sect. 3. In Sect. 4 we introduce the different models and show the results of the simulations. Conclusions are drawn in Sect. 5.

\section{Heat conduction}

The energy excange between the phases as a consequence of heat conduction is described by a heat flux $\boldsymbol{q}$. In the classical case this flux is calculated assuming a diffusion appoximation leading to the formula given by Spitzer (1962):

$\boldsymbol{q}_{\text {class }}=-\kappa \cdot \nabla T$

with the heat conduction coefficient

$\kappa=\frac{1.84 \times 10^{-5} T^{5 / 2}}{\ln \Psi} \operatorname{erg~s}^{-1} \mathrm{~K}^{-1} \mathrm{~cm}^{-1}$, where the Coulomb logarithm is

$\ln \Psi=29.7+\ln \left[\frac{T_{6, \mathrm{e}}}{\sqrt{n_{\mathrm{e}}}}\right]$,

with the electron density $n_{\mathrm{e}}$ and the electron temperature $T_{6, \mathrm{e}}$ in units of $10^{6} \mathrm{~K}$.

This description breaks down if the local temperature scaleheight falls below the mean free path of the conducting electrons. In this case the heat flux is replaced by a flux-limited form the so-called saturated heat flux (CM77):

$\left|\boldsymbol{q}_{\mathrm{sat}}\right|=5 \Phi_{\mathrm{s}} \rho c^{3}$

with the sound speed $c$ and density $\rho . \Phi_{\mathrm{S}}$ is an efficiency factor less than or of the order of unity, which embodies some uncertainties connected with the flux-limited treatment and flux suppression due to magnetic fields. This method yields results in good agreement with laser-fusion experiments (Morse \& Nielsen 1973; Mannheimer \& Klein 1975) and numerical simulations solving the Fokker-Planck equation (Khan \& Rognlien 1981; Matte \& Virmont 1982; Campbell 1984).

The heat conduction coefficient $\kappa$ is a function of the ionization state of the medium. At temperatures lower than $10^{4} \mathrm{~K}$ the ionization fraction of the gas is negligible and with this the heat conduction by electrons. Inside the cloud only conduction by neutrals may play a role for the temperature distribution. On the other hand the temperature gradient in this part of the cloud is too smooth to create a significant heat flux so that heat conduction becomes negligible. The evaporation and condensation of interstellar clouds was first investigated by Zel'dovich \& Pikel'ner (1969) and later by Penston \& Brown (1970) in a plane parallel approximation. The more realistic case of spherical clouds was examined by Graham \& Langer (1973). They solved the timeindependent energy equation including heat conduction. As a main result they concluded that clouds with radii below a critical radius suffer from evaporation while clouds with larger radii gain mass by condensation of material onto the cloud surface because radiative cooling exceeds the heat input due to heat conduction. Numerical simulations were performed by Chevalier (1975) in order to describe the evolution of interstellar clouds in young supernova remnants. Although the temperatures rose up to about $10^{7} \mathrm{~K}$ he took only the classical heat flux into account and, by this, overestimated the mass-loss rate by some orders of magnitude.

CM77 made a general study of the evaporation of spherical clouds including saturation effects. In the treatment of the saturation of nonmagnetic, nonradiative, spherical evaporation, the domain outside the cloud is broken up into three zones, each of which is treated seperately. These zones are joined by prescribed boundary conditions for the heat flux and the temperature. The heat conduction of the innermost zone which inner boundary is the cloud surface is described by the classical heat flux. For larger radii the temperature rises and the heat flux becomes saturated. CM77 treated this by abruptly changing the mathematical form of the conductivity at a well-defined saturation radius. The temperature rises steeply through the saturation zone until it reaches the value of the ambient gas. At this radius, CM77 switched back to the classical description of the heat flux. Beside a local saturation parameter $\sigma$ as the ratio of the classical to the saturated heat flux,

$\sigma=\left|\frac{\kappa}{5 \Phi_{\mathrm{s}} \rho c^{3}} \frac{\mathrm{d} T}{\mathrm{~d} r}\right|$ 


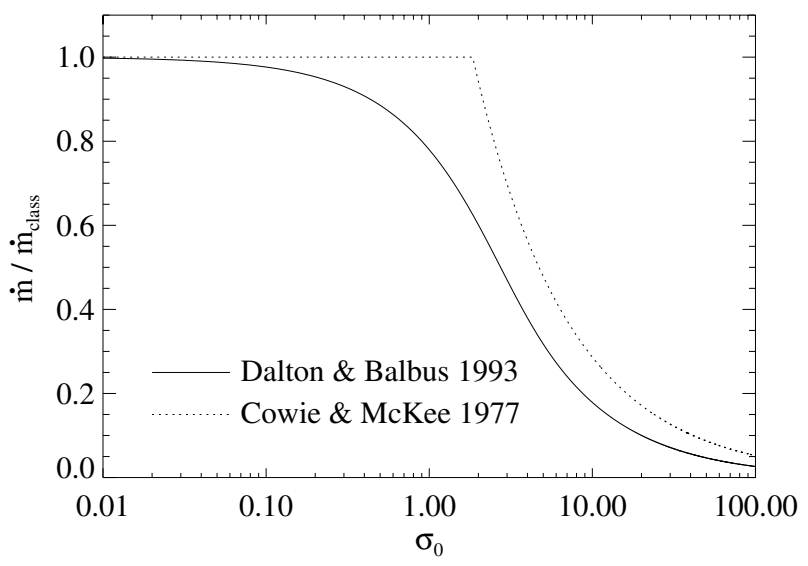

Fig. 1. Mass-loss rate $\dot{m}$ normalized to the classical value $\dot{m}_{\text {class }}$ (Eq. (7)) as a function of the global saturation parameter $\sigma_{0}$. Comparison of the rates refering to CM77 (dotted) and DB93 (solid curve).

they introduced a global saturation parameter $\sigma_{0}$

$\sigma_{0}=\frac{2 \kappa_{\mathrm{f}} T_{\mathrm{f}}}{25 \Phi_{\mathrm{s}} \rho c^{3} R}=\left(\frac{T_{\mathrm{f}}}{1.54 \times 10^{7} \mathrm{~K}}\right)^{2} \frac{1}{n_{\mathrm{f}} \Phi_{\mathrm{s}} R_{\mathrm{pc}}}$

in order to ascertain the influence of saturation effects only by knowing the cloud radius $R$ or $R_{\mathrm{pc}}$ the same in parsec and the heat conduction coefficient, the temperature and particle density of the ambient medium $\kappa_{\mathrm{f}}, T_{\mathrm{f}}$ and $n_{\mathrm{f}}$. For $\sigma_{0} \leq 1$ the mass-loss is given by

$\dot{m}_{\text {class }}=\frac{16 \pi \mu \kappa_{\mathrm{f}} R}{25 k}$

where $k$ is Boltzmann's constant and $\mu$ is the mean molecular weight of the ambient plasma. For larger values of $\sigma_{0}$ the mass loss is a decreasing function of $\sigma_{0}$ (see Fig. 1).

In a following paper McKee \& Cowie (1977) analysed the influence of radiative cooling on the evaporation rate. They found out that the additional heat input due to heat conduction is compensated by radiative cooling for $\sigma_{0}<0.027 / \Phi_{\mathrm{s}}$. Also in this study the authors devided the cloud environment into three zones where the physics are described in different ways. In the inner and the outer zone radiative losses are larger than the spherical divergence of the heat flux, while in the zone between the opposite is true and radiative losses are neglegted. Because of the fact that only the classical heat flux is considered, this approach can be only considered as a very crude estimation for the mass loss rate. Saturation reduces the energy input into the cloud so that radiation cooling can exceed this additional heat input.

Begelman \& McKee (1990) investigated the evolution of a two-phase medium consisting of clouds embedded in a hot plasma. They showed that heating and cooling processes dominate the hot phase and heat conduction can be neglected if the length scales of the relevant structures are larger than a critical lenght, the so-called Field length:

$\lambda_{\mathrm{F}} \equiv\left[\frac{\kappa T}{n^{2} \mathcal{L}_{\mathrm{M}}}\right]^{1 / 2}$

where $\mathcal{L}_{\mathrm{M}}$ defines the maximum heating or cooling rate. The Field length is the maximum length scale on which energy transport by heat conduction is effective. So the temperature distribution of structures with $r \ll \lambda_{\mathrm{F}}$ that are embedded in the hot plasma is dominated by heat conduction while this process can be neglected for $r \gg \lambda_{\text {F }}$. For an isolated cloud they concluded

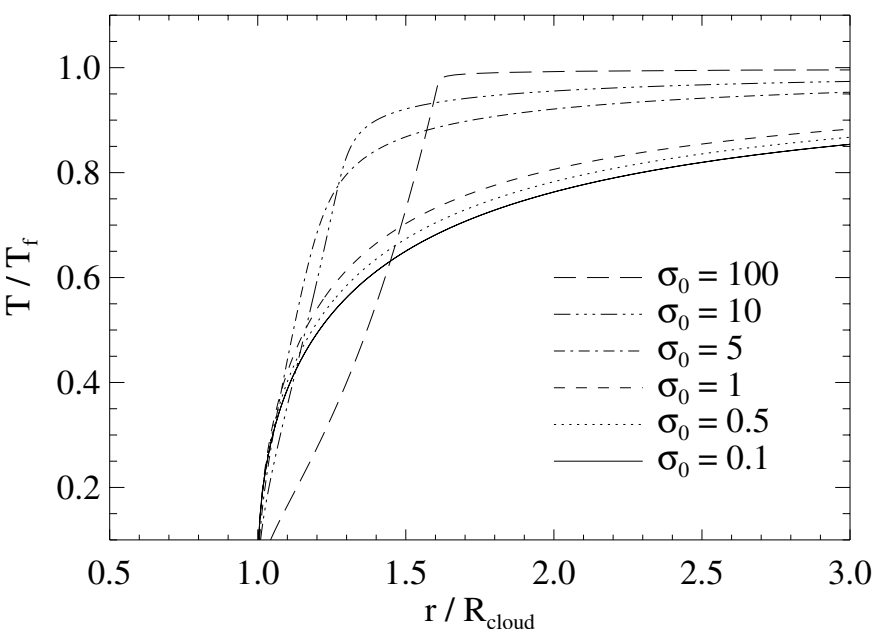

Fig. 2. Temperature profiles for different values of $\sigma_{0}$ derived from DB93.

that the classical mass-loss rate is valid for clouds with $R \ll \lambda_{\mathrm{F}}$ while condensation occurs for $R \approx 0.24-0.36 \lambda_{\mathrm{F}}$.

DB93 recovered the considerations of CM77 but this time used an effective heat flux that is the harmonic mean of the classical and the saturated flux in order to get a smooth transition between both regimes:

$q_{\mathrm{eff}}=-\frac{\kappa}{1+\sigma} \frac{\mathrm{d} T}{\mathrm{~d} r}$

Also in this description the time-independent energy equation can be solved analytically. This leads to the temperature distribution outside the cloud and also to the mass-loss rate as a function of $\sigma_{0}$. The mass loss rate is compared with the one derived by CM77 in Fig. 1. The temperature distributions calculated by the formulae of DB93 for different values of $\sigma_{0}$ (Fig. 2) reflect the two different mathematical descriptions of the heat flux. The solutions for the classical flux $\left(\sigma_{0} \leq 1\right)$ show a steep temperature increase at the edge of the cloud and a decreasing gradient for larger $r$. For large $\sigma_{0}$ the curvature is positive near the cloud surface so that $T_{\mathrm{f}}$ is reached at smaller $r$ than in the classical case.

Because only the energy equation is solved in CM77 as well as in DB93, dynamical effects like mixing of the cold cloud material with the hot plasma are not considered. On the other hand, it must be expected that the cloud material that is detached from the cloud surface is still cooler than the ambient plasma because only a slight rise in temperature of the cloud material leads to its overpressure that decouples the material from the cloud's surface. This will influence the temperature distribution near the surface and, by this, alter the mass-loss rate accordingly. Therefore, the hydrodynamical treatment is essential in order to explore to what extent the mass flow by evaporation and condensation and further gas-phase mixing changes the physical conditions around the interface.

\section{Hydrodynamical treatment}

The spherical geometry of the cloud suggests to use a one dimensional spherical symmetric hydro-code. With a limitation to only one spatial dimension it is impossible to follow the growth of instabilities that are expected when cold dense gas is accelerated into warm rarefied gas. Therefore the evolution of clouds in a hot plasma is studied by two-dimensional hydrodynamical 
Table 1. Model parameters and physical processes considered in the various simulations. For all models: $R_{\text {cloud }}=41 \mathrm{pc}, T_{\mathrm{f}}=5.6 \times 10^{6} \mathrm{~K}$, $n_{\mathrm{f}}=6.6 \times 10^{-4} \mathrm{~cm}^{-1}, \sigma_{0}=4.88$.

\begin{tabular}{|c|c|c|c|c|c|c|c|c|}
\hline \multirow[t]{2}{*}{ Model } & \multirow{2}{*}{$\begin{array}{l}\text { Numerical grid } \\
\left(\text { cell }^{2}\right)\end{array}$} & \multirow{2}{*}{$\begin{array}{l}\text { Resolution } \\
\left(\mathrm{pc} \text { cell }{ }^{-1}\right)\end{array}$} & \multirow{2}{*}{$\begin{array}{l}\text { Density and temperature } \\
\text { profile }\end{array}$} & \multicolumn{2}{|c|}{ Heat conduction } & \multirow{2}{*}{$\begin{array}{l}\text { Self- } \\
\text { gravity }\end{array}$} & \multirow{2}{*}{$\begin{array}{l}\text { Heating/ } \\
\text { cooling }\end{array}$} & \multirow{2}{*}{$\begin{array}{l}\text { Mass } \\
\left(M_{\odot}\right)\end{array}$} \\
\hline & & & & class. & class. \& sat. & & & \\
\hline R1a & $800 \times 400$ & 1 & homogen. & + & - & - & - & $3.4 \times 10^{4}$ \\
\hline R1b & $800 \times 400$ & 0.5 & homogen. & + & - & - & - & $3.4 \times 10^{4}$ \\
\hline $\mathrm{R} 2 \mathrm{a}$ & $200 \times 100$ & 1 & homogen. & - & + & - & - & $3.4 \times 10^{4}$ \\
\hline $\mathrm{R} 2 \mathrm{~b}$ & $1000 \times 500$ & 1 & homogen. & - & + & - & - & $3.4 \times 10^{4}$ \\
\hline $\mathrm{R} 2 \mathrm{c}$ & $900 \times 450$ & 0.33 & homogen. & - & + & - & - & $3.4 \times 10^{4}$ \\
\hline R3 & $200 \times 100$ & 1 & core-halo & - & + & - & - & $6.4 \times 10^{4}$ \\
\hline R4 & $200 \times 100$ & 1 & core-halo & - & + & + & - & $6.4 \times 10^{4}$ \\
\hline R5 & $200 \times 100$ & 1 & core-halo & - & + & + & + & $6.4 \times 10^{4}$ \\
\hline
\end{tabular}

simulations. The Eulerian equations are solved on a rectangular cylindrically symmetric "staggered grid" which is of second order in space and based on the prescription of Rozyczka (1985). This code was extensively tested and used by different authors (e.g. Yorke \& Welz 1996). The grid parameters, the resolution, the density/temperature profile of the initial cloud model and the considered physical processes are listed in Table 1 for the different models.

The different setups for the homogeneous clouds are chosen in order to test the boundary conditions (model R2b) and the influence of resolution on the mass-loss rate (model R1b, R2c).

The boundary conditions at the upper, the left-hand and the right-hand sides are semi-permeable to allow for an outflow of gas from the computational domain. The physical parameters at the lower boundary, the symmetry axis, are mirrored. In order to trace the condensation of the hot plasma onto the cloud a new quantity "colour" is introduced that is set in each cell to the density fraction of hot ISM. At the beginning only the cells around the cloud possess a non-zero "colour" of value unity. During the calculation this quantity is advected like the others as e.g. mass or energy density. For the advection we use the monotonic transport (van Leer 1977). The Poisson equation for self-gravity was solved for the corresponding model at each tenth timestep because significant density changes of the cloud structure happen on a much larger timescale than the dynamical one. The energy equation includes heating, cooling and heat conduction, according to what physical process is included in the model:

$\frac{\partial e}{\partial t}+\boldsymbol{\nabla} \cdot(e \boldsymbol{v})=-P \boldsymbol{\nabla} \cdot \boldsymbol{v}+\Gamma-\Lambda-\boldsymbol{\nabla} \cdot \boldsymbol{q}$.

Here $e$ denotes the energy density, $v$ the velocity, $P$ the pressure, $\Gamma$ the heating rate, $\Lambda$ the cooling rate and $\boldsymbol{q}$ the heat flux. The equation of state for an ideal gas is assumed to be valid:

$P=(\gamma-1) e \quad$ with $\quad \gamma=5 / 3$.

The used cooling function assumes collisional ionisation equilibrium and is a combination of the function introduced by Böhringer \& Hensler (1989) for $T>10^{4} \mathrm{~K}$ and solar metallicity and by Dalgarno \& McCray (1972) for the lower temperature regime. The heating function considers cosmic rays (Black 1987), X-rays and the photoelectric effect on dust grains (de Jong 1977; de Jong et al. 1980). This detailed description of heating and cooling processes cannot be incorporated in analytical work like in CM77 that approximates the cooling function by four power-law segments. For temperatures above $10^{4} \mathrm{~K}$ we used a more up-to-date description than CM77 who derived the cooling function in this temperature regime from Raymond et al. (1976). Nevertheless, the cooling function used in our simulations is similar to the one used by CM77.
The heat flux is calculated by taking both, the classical and the saturated flux into account. In order to apply a smooth transition between classical and saturated regime we use the analytical form by Slavin \& Cox (1992)

$\boldsymbol{q}=\left|\boldsymbol{q}_{\text {sat }}\right|\left(1-\exp \left[-\frac{\boldsymbol{q}_{\text {class }}}{\left|\boldsymbol{q}_{\text {sat }}\right|}\right]\right)$

This guarantees that the smaller flux is taken if both differ significantly. The heat flux due to electron diffusion is calculated separately using an implicit method which follows a scheme introduced by Crank \& Nicolson (1947) and Juncosa \& Young (1971). To couple the two directions in space the method of fractional steps by Yanenko (1971) is used. A detailed description of the implementation as well as a numerical test of the heat conduction code can be found in the online appendix.

The simulated clouds are embedded in a hot plasma with a given temperature $T_{\mathrm{f}}=5.6 \times 10^{6} \mathrm{~K}$ and particle density $n_{\mathrm{f}}=$ $6.6 \times 10^{-4} \mathrm{~g} \mathrm{~cm}^{-3}$. The most realistic cloud model numbered as R5 is generated for hydrostatic and thermal equilibrium under the constraint of spherical symmetry:

$\rho \boldsymbol{\nabla} \cdot \Phi=-\boldsymbol{\nabla} \cdot P$

$\Gamma(\boldsymbol{r})=\Lambda(\boldsymbol{r})$

$\Phi(r)$ is the gravitational potential. The density and temperature profile of the cloud is then calculated by integrating Eqs. (13) and (14) from inside-out using the core temperature of the cloud as a boundary condition and truncating the cloud's outermost border where the energy density of the cloud reaches the value of the hot plasma $e_{\mathrm{f}}$. This condition is fulfilled for a radius of $R_{\mathrm{cld}}=$ $41 \mathrm{pc}$. The density distribution we get in this way shows a clear core-halo structure with a tenuous rim and a pronounced central core (Figs. 3 and 4) similar to observed interstellar clouds.

This cloud radius together with the hot plasma parameters leads to a global saturation parameter of $\sigma_{0}=4.88\left(\Phi_{s}=1\right)$ for which saturation of the heat flux is expected so that the classical mass-loss rate should be reduced for saturation effects according to CM77 by $51 \%$ to a value of $5.6 \times 10^{-4} M_{\odot} \mathrm{yr}^{-1}$ or according to DB93 by $67 \%$ to a value of $3.7 \times 10^{-4} M_{\odot} \mathrm{yr}^{-1}$, respectively.

In order to be close to the analytical considerations also a cloud with homogeneous density distribution is treated, denominated as R1. With the same surface density and temperature as the stratified clouds it fulfills the condition of pressure equilibrium with the ambient medium. The heat conduction is here implied only classically. Self-gravity, heating or cooling are neglected. For models R2 the density and temperature structures are not altered but the physics are extended in a way, that now the effective heat flux is taken into account. These models can be used for a comparison with the analytical predictions of CM77 


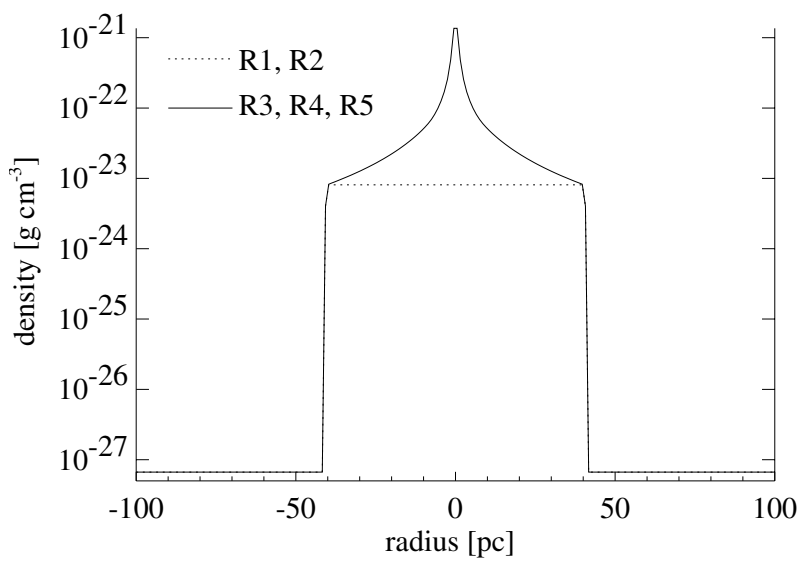

Fig. 3. Density profiles for the models with homogeneous density distribution (dotted) and for those with pronounced central peak (solid line).

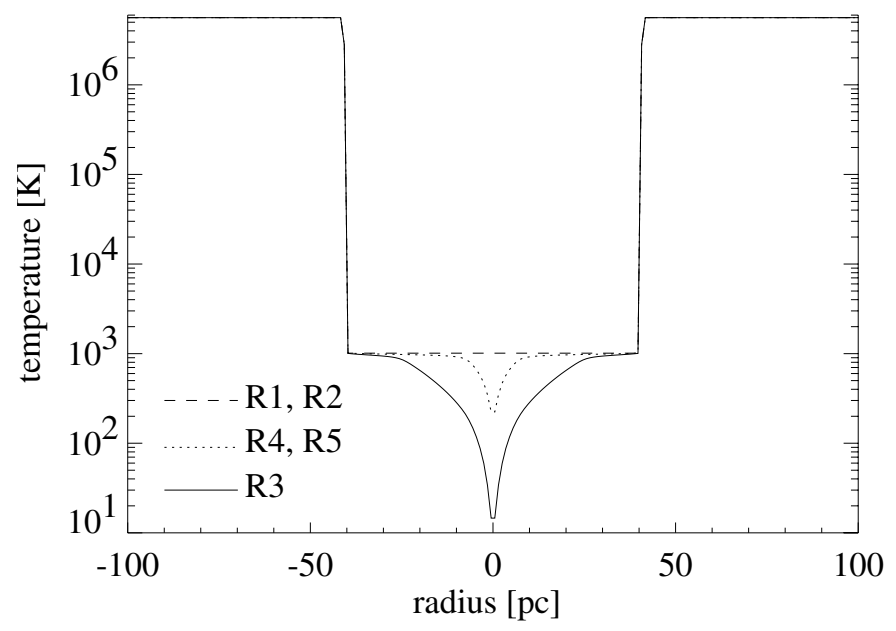

Fig. 4. Temperature profiles for the various models. Models R1 and R2 show a homogeneous distribution in order to fulfil pressure equilibrium with a homogeneous density distribution. Pressure equilibrium is necessary also for model R3 with a pronounced central density peak. The temperature profile for model R4 is calculated for hydrostatic equilibrium, model R5 has to fulfil thermal equilibrium as well. This does not alter the temperature distribution because of the chosen density profile.

or DB93. For comparison reasons of all model clouds (also of the homogeneous clouds) we fixed the radius to that of the R5 model, namely, $41 \mathrm{pc}$ (Figs. 3 and 4) and, by this, the value of $\sigma_{0}$.

In a more advanced model (R3) the density distribution is then modified according to a core-halo structure, while the temperature distribution has also to be changed in order to guarantee pressure equilibrium (Fig. 4). The mathematical form of the heat conduction process remains the same. As the further improvement for model R4 we apply the same density structure as in model R3 but take now self-gravity into account. The temperature profile has to change accordingly in order to serve for hydrostatic equilibrium. And finally, as mentioned at the beginning of this part, R5 is developed as the most advanced model implying also thermal equilibrium.

\section{Models}

Here we present the evolution of the five generally different models and their variations with respect to the numerical resolution.

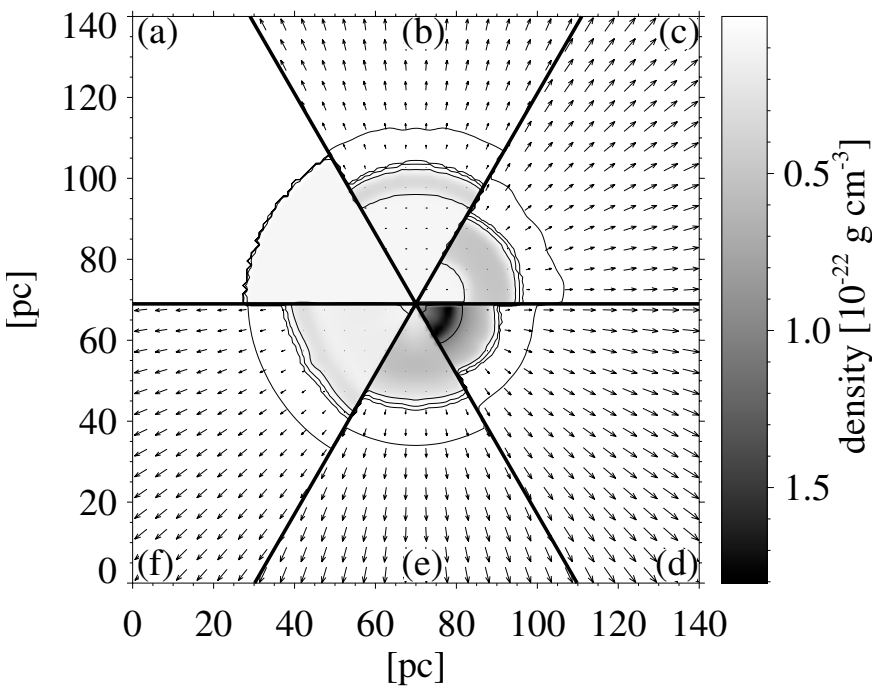

Fig. 5. Evolution of the density distribution of model R1a after $0 \mathrm{Myr}$ a), $1 \mathrm{Myr}$ b), $2 \mathrm{Myr} \mathbf{c}$ ), $3 \mathrm{Myr}$ d), $4 \mathrm{Myr}$ e) and $5 \mathrm{Myr} \mathbf{f}$ ). Lines of equal density are drawn between $\rho=10^{-22}$ and $10^{-26} \mathrm{~g} \mathrm{~cm}^{-3}$ in steps of $1 \mathrm{dex}$.

\subsection{Model R1a-R1b}

Although the global saturation parameter $\sigma_{0}$ suggests that saturation effects in the heat flux are to be expected, this first simulation is performed by only using the classical form of the heat flux. This is done in order to investigate the consequence of this simple approach on the mass-loss rate. The model starts with a homogeneous cloud of $\rho=8 \times 10^{-24} \mathrm{~g} \mathrm{~cm}^{-3}$ and a spatially constant temperature of $T=1000 \mathrm{~K}$. The temporal evolution of the density distribution is shown in Fig. 5. The velocity field visualized by arrows in regions where $\rho<10^{-25} \mathrm{~g} \mathrm{~cm}^{-3}$ is directed away from the cloud during the whole simulation. This is a clear indication for mass loss, while the inner parts of the cloud begin to collapse. The trigger for this process is the huge heat input at the cloud edge due to heat conduction, that rises the temperature there and leads to an overpressure with a two-fold consequence: first, it pushes material away from the surface into the ICM whereby the evaporated material is by far not as hot as the ICM when it decouples from the cloud. So the resulting temperature distribution outside the cloud is a rising function with the cloud distance (see Fig. 6). Secondly, the evaporated material generates a repulsion that leads to a preassure wave running through the cloud and further to a pulsation of the cloud as a whole. The pulsation timescale corresponds very well to the dynamical timescale of the cloud which is the sound crossing time. One has to keep in mind that the oscillations are excited artificially by the switch-on of the numerical simulation.

After some $10^{5}$ years a quasistatic temperature distribution has formed (see Fig. 6). In this figure also the pulsation of the cloud can be revealed. A comparison of $T(r)$ at the end of the calculation with an analytically derived distribution for $\sigma_{0}<1$ (see Fig. 2) shows a qualitatively good agreement. The steep rise in temperature at the cloud surface and a smooth asymptotic behaviour for large $r$ is typical for calculations with only the classical heat flux.

The mass-loss rate is calculated far away from the cloud at a distance of $95 \mathrm{pc}$ using

$\dot{m}=4 \pi r^{2} \rho(r) v(r)$.

Positive values of $\dot{m}$ stand for mass loss or evaporation of the cloud while negative values of $\dot{m}$ indicate condensation of hot 


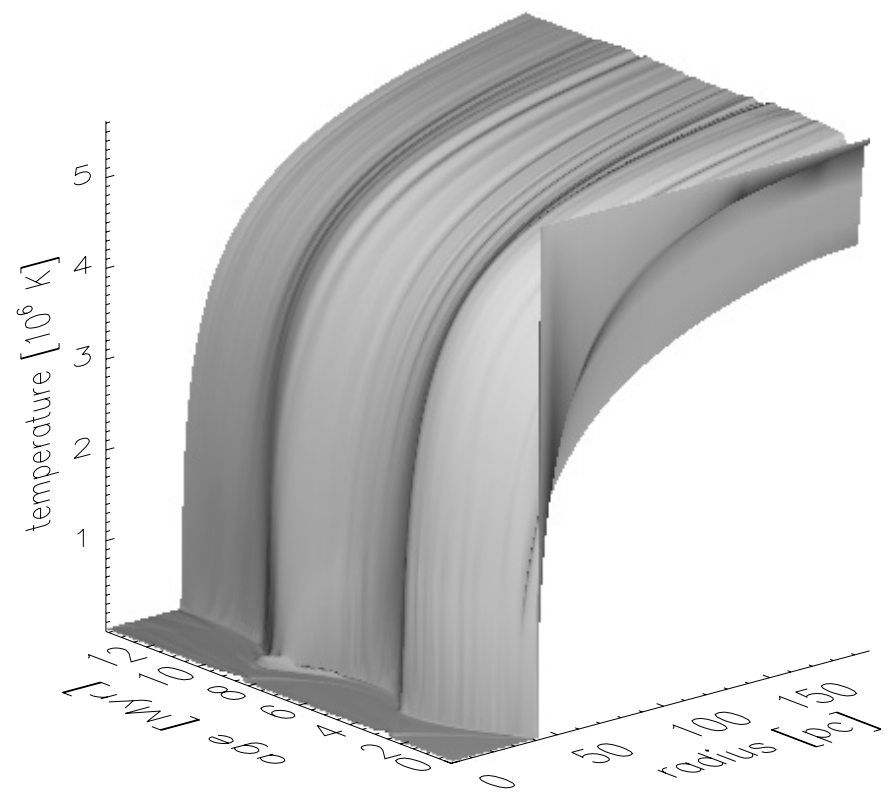

Fig. 6. Evolution of the temperature profile of model R1. The course is in good agreement with an analytically derived distribution for $\sigma_{0}<1$ that takes only the classical heat flux into account.

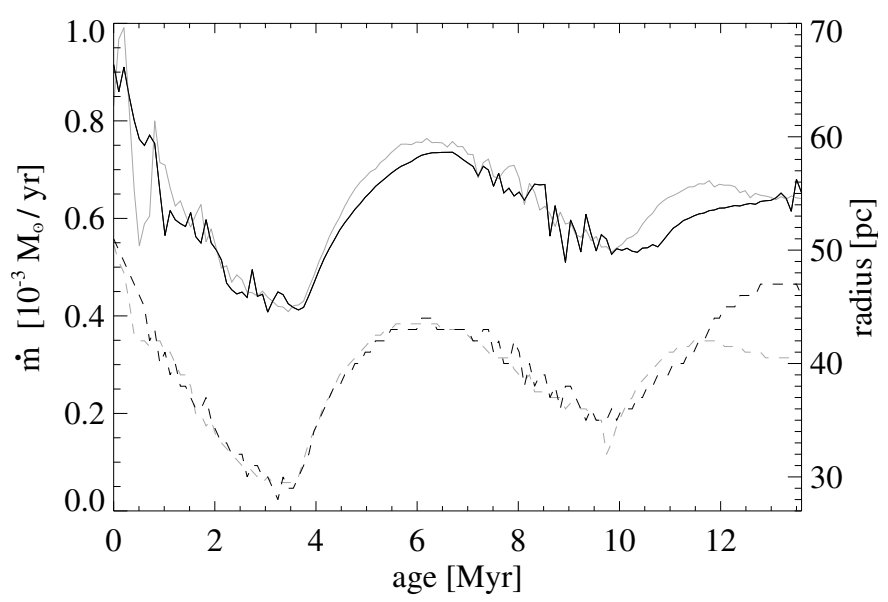

Fig. 7. Mass-loss rate for the models $1 \mathrm{a}$ (black solid curve) and $1 \mathrm{~b}$ (grey solid curve) compared with their cloud radius (lower black/grey dashed lines).

ISM onto the cloud. During the whole simulation evaporation occurs (see Fig. 7) and its strength is well correlated with the radius of the cloud as expected from Eq. (7). $\dot{m}$ according to CM77 or DB93 requires a value of about $1 \times 10^{-3} M_{\odot} \mathrm{yr}^{-1}$. This is in relatively good agreement with the value derived from the simulations $\left(\dot{m} \approx 0.8 \times 10^{-3} M_{\odot} \mathrm{yr}^{-1}\right.$; see Table 2$)$. At the beginning $\dot{m}$ represents the analytical value, and this more precisely for the spatially higher resolved simulation, what demonstrates the accuracy of our treatment. The model with higher resolution shows no significant differences in its structure or evolution. Also the mass-loss rate is compareable. This is an indication that we can handle heat condution also in the low resolution case so that no time consuming highly resolved simulations are necessary as long as one is not interested in surface structural effects.

The limitation of heat conduction to the classical case leads to a very large mass-loss rate in analytical studies as well as in numerical simulations. Due to the enormous energy input the evaporated material is accelerated to velocities near sonic speed.

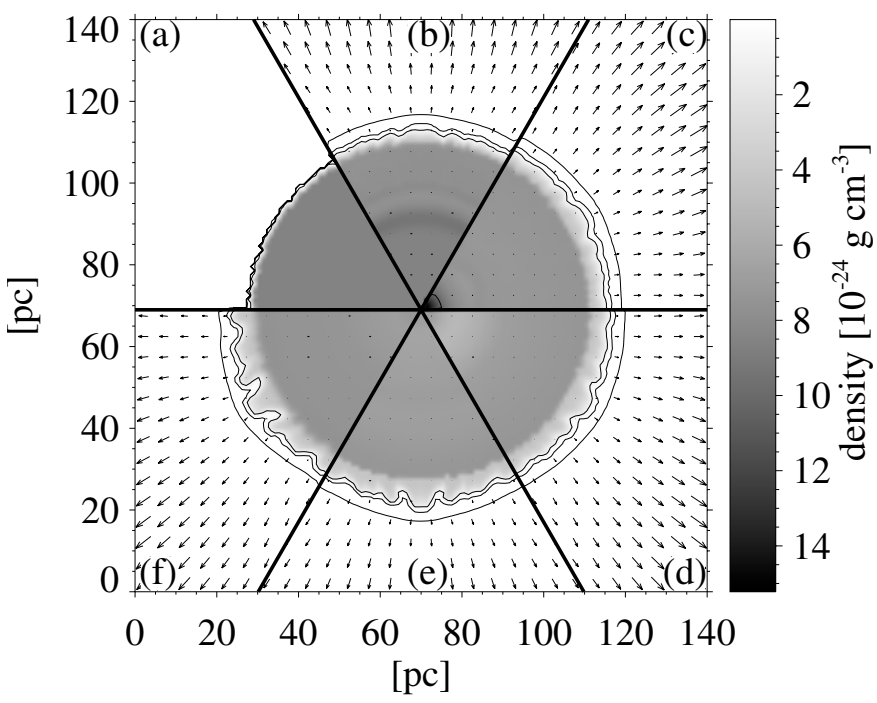

Fig. 8. Evolution of the density distribution of model R2a after $0 \mathrm{Myr}$ a), $5 \mathrm{Myr}$ b), $10 \mathrm{Myr} \mathbf{c}$ ), $15 \mathrm{Myr}$ d), $20 \mathrm{Myr}$ e) and $25 \mathrm{Myr} \mathbf{f}$ ). Lines of equal density are drawn between $\rho=10^{-22}$ and $10^{-26} \mathrm{~g} \mathrm{~cm}^{-3}$ in steps of 1 dex.

\subsection{Model R2a-R2c}

These models differ from $\mathrm{R} 1$ in that saturation effects are now taken into account. The density and temperature structure of the initial model is the same as in R1. The evolution in time of the density distribution is shown in Fig. 8. Because of the limitation of the energy transport due to the saturated heat flux, also the energy input and with this the rise in temperature at the cloud surface is reduced. The resulting overpressure at the cloud rim is lower than in model R1. Nevertheless, a pressure wave that runs through the cloud and causes density fluctuations inside the cloud is triggered by the evaporated material (see innermost denser shell in Fig. 8b). In contrast to model R1, here no largescale oscillations occur.

Also in this model cloud material is evaporated from the surface as a consequence of the additional heat input at the cloud surface due to heat conduction. Unlike model R1 the overpressure is not large enough to blow this material far and fast into space but it is mixed with the hot ICM and forms a transition layer around the cloud. The extension of this layer is visible in Fig. 8 by the growth of the distances between iso-density lines at the edge of the cloud. As time procedes Rayleigh-Taylor (RT) instability grows in the transition layer because warm and dense material is continuously accelerated into the hot rarefied plasma. The linear growth timescale for RT instability is $\tau_{R T}=\sqrt{\frac{\lambda}{a}}$ where $\lambda$ is the size of the initial perturbation which lies in the range of the cell size and $a$ is the effective acceleration. Assuming that the evaporated cloud material is accelerated to a value of 0.05 Mach over a distance of $3 \mathrm{pc}$ in the transition layer, $\tau_{R T}$ amounts to $0.8 \mathrm{Myr}$ which is in good agreement with the value derived from the simulation. RT instability would be also expected to arise in models R1, because the conditions for instability are analytically fulfilled. However, the strong pulsations in this model are responsible to prevent its growth: phases in which RT instability is forced, i.e. when the cloud surface is accelerated outwards, are too short to allow its discernible growth.

The extension of the transition layer is better visualized in Fig. 9 where the density profiles of the different models R2a$\mathrm{R} 2 \mathrm{c}$ are plotted as a function of the radius at time $t=30 \mathrm{Myr}$. The transition layer is clearly visible between $40 \mathrm{pc}<r<48 \mathrm{pc}$. 


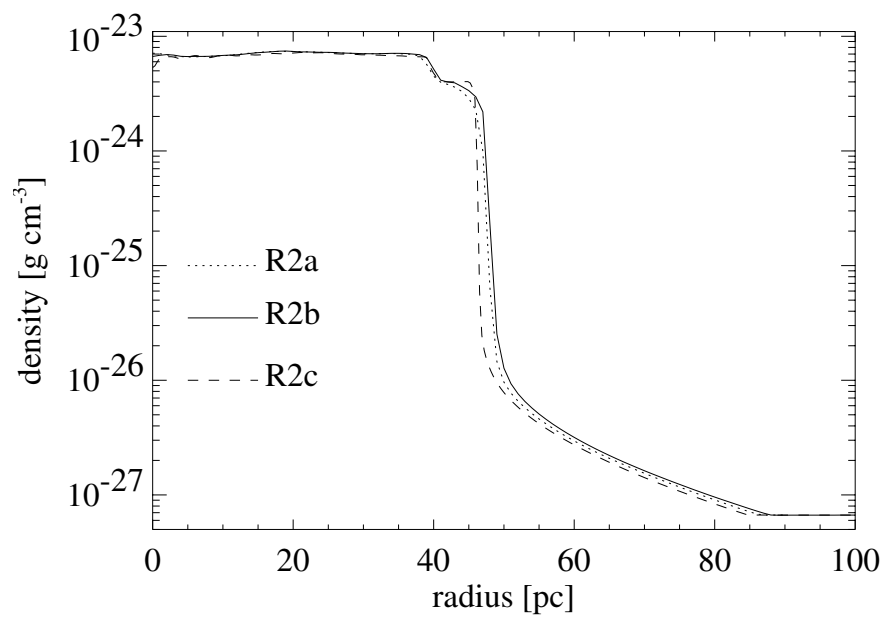

Fig. 9. Density profiles of the models R2a-R2c at a time $t=30 \mathrm{Myr}$. The transition region for $40 \mathrm{pc}<r<48 \mathrm{pc}$ is clearly visible.

The outer edge of the layer cannot be sharply defined. Also for larger radii up to $85 \mathrm{pc}$ significant amounts of cloud material can be found.

The results of the different R2 models show only weak dependencies on resolution or boundary conditions. In particular, the plateau between $40 \mathrm{pc}$ and $45 \mathrm{pc}$ in all R2 models in Fig. 9 remains the same and reflects the regime where RT instability is excited. The fluctuations inside the cloud result from the pressure waves triggered by the repulsion of the evaporated material. The temperature inside the transition zone is rising with radius and has values of $T \approx 1.5 \times 10^{3} \mathrm{~K}$ at the inner boundary $(r=40 \mathrm{pc})$ and reaches $T \approx 10^{5} \mathrm{~K}$ at $r=48 \mathrm{pc}$. Because the density structure of the layer runs oppositely to the temperature structure, the material in the transition zone can exist almost in pressure equilibrium. Nevertheless, the remaining slight pressure gradient at the outer boundary leads to the observed evaporation. Like in the previous model the velocity field is directed outwards and indicates evaporation. In contrast to model $\mathrm{R} 1$, the maximum speed of the lost material is subsonic with only $0.05 \mathrm{Mach}\left(\approx 21 \mathrm{~km} \mathrm{~s}^{-1}\right)$. This mass-loss rate amounts to $\dot{m} \approx 1.4 \times 10^{-5} M_{\odot} \mathrm{yr}^{-1}$ (see Fig. 13) and is therefore only $1 / 40$ of the analytically predicted one and agrees at best for a model with $\sigma_{0} \approx 100$. We can show that the low Mach number is caused by the energy loss of the expanding (and evaporating) shell due to $p \cdot d V$ work against the external pressure. From our R2 model one can derive that the specific thermal energy is reduced by a factor of almost 500. This value can be derived from the density and temperature values at the cloud edge (at $46 \mathrm{pc}$ ) and at $r=67 \mathrm{pc}$ where $T_{\mathrm{f}}=5.6 \times 10^{6} \mathrm{~K}$ is reached. The density contrast amounts to $3 \times 10^{3}$ (see Fig. 9), the temperature contrast to 0.16 (see Fig. 10). Since the analytical consideration by CM77 cannot account for this physical process, the evaporation in their model remains at sound speed. The slight differences in the simulations with different spatial resolutions and grid extension are not significant so that simulations with a resolution of $1 \mathrm{pc}$ cell ${ }^{-1}$ are reliable. A comparison of the temperature profile of the simulation (see Fig. 10) with an analytical one for $\sigma_{0}=4.8$ yields no good result whereas a theoretical profile for $\sigma_{0}=100$ fits the simulated model very well again. Conclusively, the temperature profile is the most important factor for the value of the evaporation rate and, on the other hand, results from the local heat flux that is a function of the density in the case of saturated heat conduction. With the formation of the transition zone at the edge of

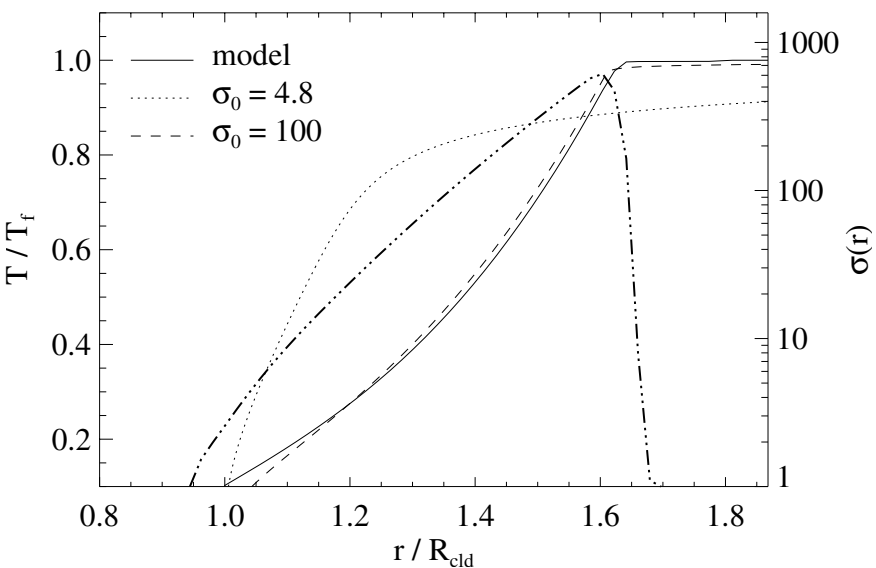

Fig. 10. Comparison of different temperature profiles between the model R2a after $62 \mathrm{Myr}$ (solid curve) and theoretical profiles of constant $\sigma_{0}: 4.8$ (dotted), $\sigma_{0}=100$ (dashed). Additionally the local saturation parameter $\sigma(r)$ deduced from the simulation is plotted (dashed-dotted). The temperature is normalized to $T_{\mathrm{f}}=5.5 \times 10^{6} \mathrm{~K}$.

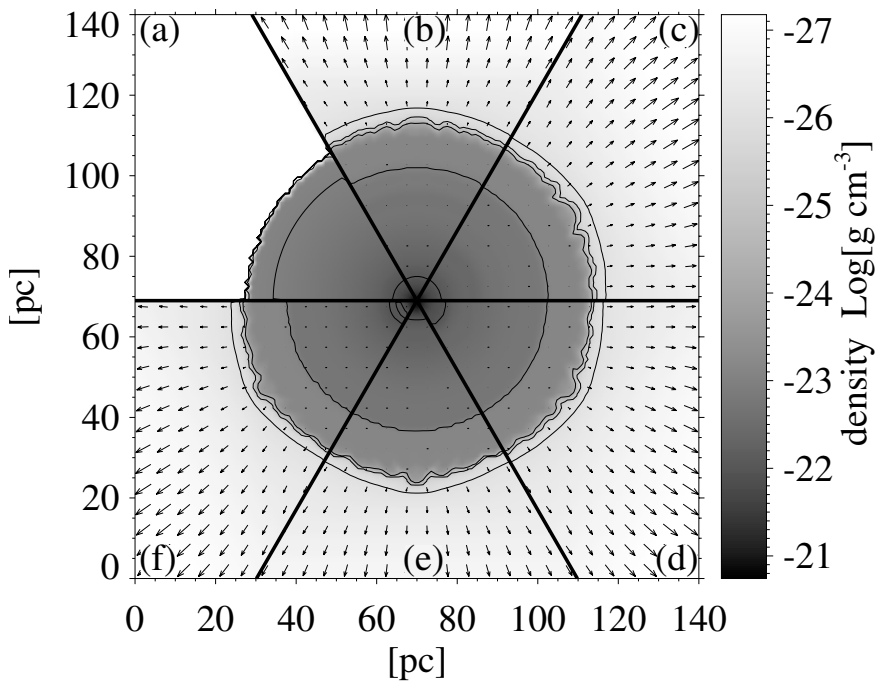

Fig. 11. Evolution of the density distribution of model R3 after $0 \mathrm{Myr}$ a), $5 \mathrm{Myr}$ b), $10 \mathrm{Myr}$ c), $15 \mathrm{Myr}$ d), $20 \mathrm{Myr}$ e) and $25 \mathrm{Myr} \mathbf{f})$. Lines of equal density are drawn between $\rho=10^{-22}$ and $10^{-26} \mathrm{~g} \mathrm{~cm}^{-3}$ in steps of 1 dex.

the cloud the density distribution has changed from the initial model and so does the evaporation rate.

\subsection{Model R3}

High-resolution observations of interstellar clouds, e.g. HVCs, show that most of them consist of an inhomogeneous gas and dust distribution with dense cores and rarefied envelopes. In order to analyze the influence of the heat conduction process on more realistic core-halo structures, the following cloud model consists of a pronounced core without self-gravity or heating and cooling. The temperature distribution of the initial model was chosen to account for pressure equilibrium. In the core region with some 10 particles $\mathrm{cm}^{-3}$ the temperature drops to about $10 \mathrm{~K}$. Towards the edge it rises to $T=300 \mathrm{~K}$ at $r=10 \mathrm{pc}$ and $T=1000 \mathrm{~K}$ at $r=30 \mathrm{pc}$. Because the heat flux is very small in the inner regions as a consequence of the low temperature, its influence will be limited to the cloud edge. The evolution of the density distribution is shown in Fig. 11. Similar to model 


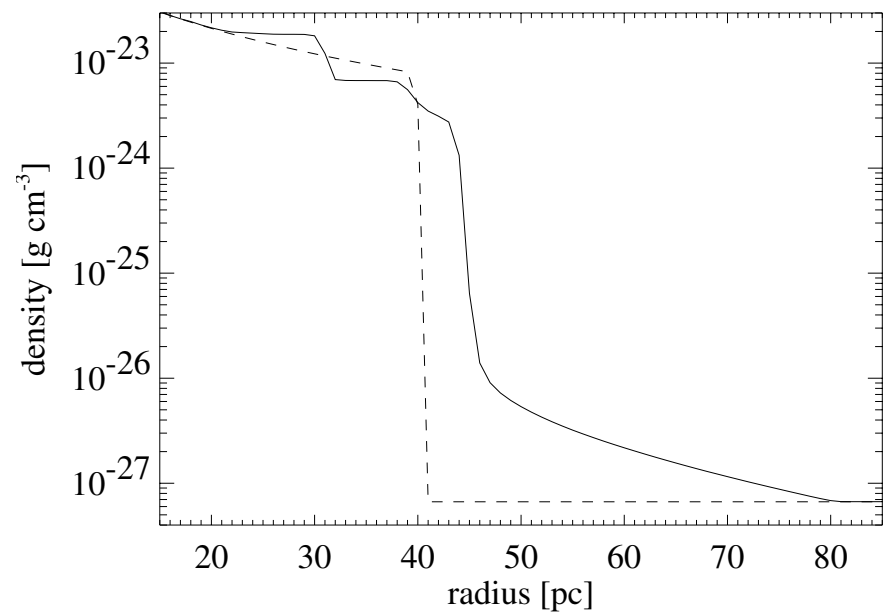

Fig. 12. Density profile of the model R3 at a time $t=37 \mathrm{Myr}$ (solid curve) in comparison with the initial model (dashed).

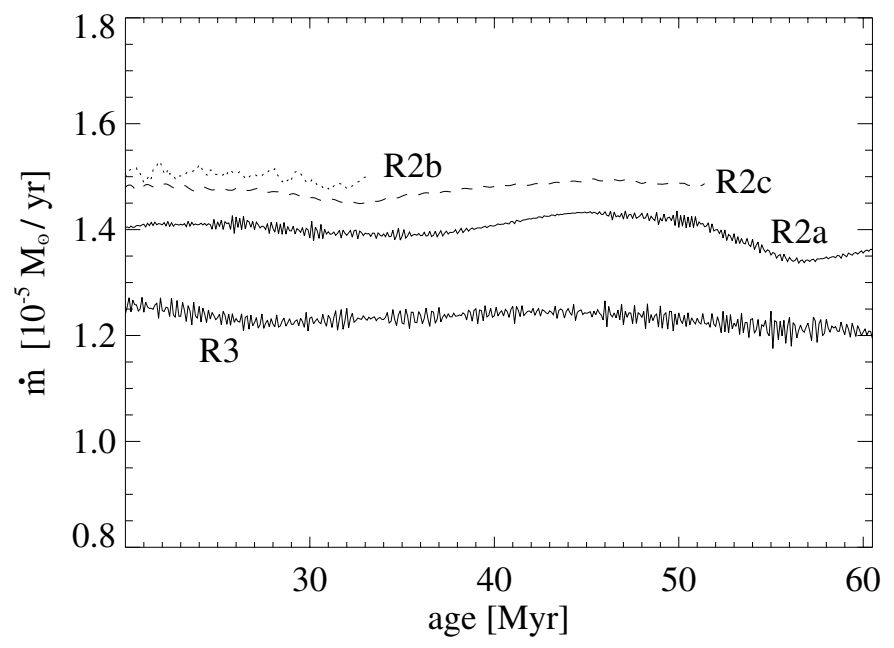

Fig. 13. Evaportion rates for the models $R 2 a-R 2 c$ and $R 3$.

$\mathrm{R} 2$, a transtion region forms that is visible by the growth of the separation of density isolines at the edge of the cloud. Also the growth of RT instabilies is discernible.

In order to analyze the evolution at the cloud rim, a density profile at $t=37 \mathrm{Myr}$ is plotted in Fig. 12 and compared with the initial model. Clearly visible is the transition region with similar expansion like model R2. This expansion excites sound waves travelling inwards, by this, dissipating energy, and rising the temperature by about $150 \mathrm{~K}$ in the region $30 \mathrm{pc}<r<40 \mathrm{pc}$ whereas the core region remains nearly unaffected. The cloud's density distribution becomes quasistatic after the redistribution of the density so that a new pressure equilibrium is reached. The cloud structure is now comparable to model R2 except the fact, that the innermost part with $r<30$ pc is almost inert and characterized by its huge density and low temperature. Also the density distribution inside the transition layer is comparable to the one of model R2 (see Figs. 9 and 12 for comparison). Because the evaporation rate is strongly determined by the processes in this transition layer, it is expected, that the mass-loss rate of both models should be the same (see Fig. 13). The reason for the slightly lower evaporation rate for model R3 can be found by the fact, that evaporated material can be provided only from the homogeneous part of the cloud within $30 \mathrm{pc}<r<40 \mathrm{pc}$ while the innermost part of the cloud is inert against heat conduction.

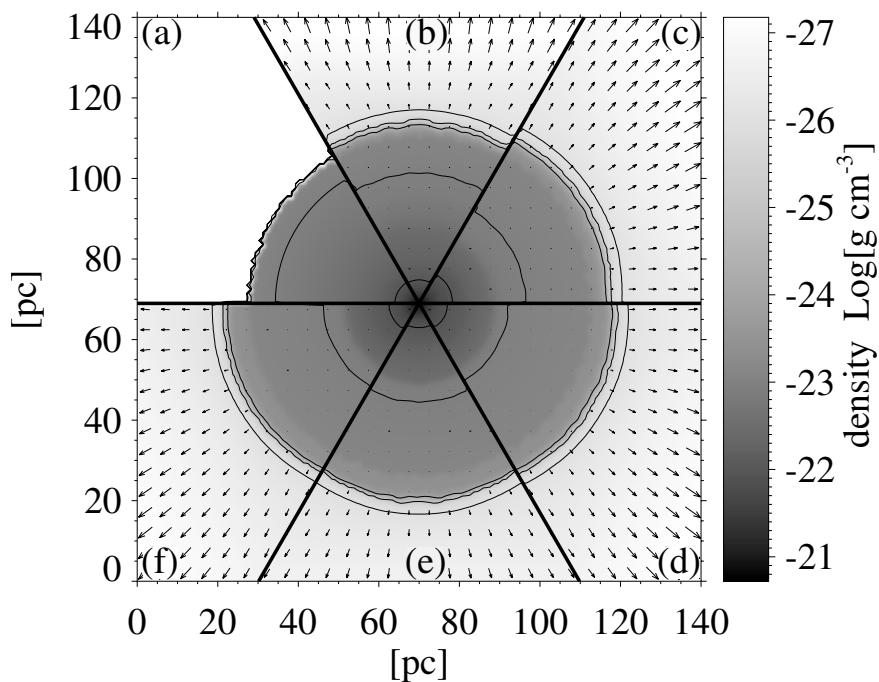

Fig. 14. Evolution of the density distribution of model R4 after $0 \mathrm{Myr}$ a), $5 \mathrm{Myr}$ b), $10 \mathrm{Myr} \mathbf{c}), 15 \mathrm{Myr}$ d), $20 \mathrm{Myr}$ e) and $25 \mathrm{Myr} \mathbf{f})$. Lines of equal density are drawn between $\rho=10^{-22}$ and $10^{-26} \mathrm{~g} \mathrm{~cm}^{-3}$ in steps of 1 dex.

Conclusively no significant differences can be found in the evolution between a multi-phase cloud and a homogeneous cloud.

\subsection{Model R4}

Clouds like those simulated in model R3 are massive enough so that self-gravity is no longer negligible. Model R4 was therefore calculated with self-gravity by solving the Poisson equation for the corresponding density distribution. The temperature of the initial model was set for hydrostatic equilibrium. The dense core has therefore to achieve a higher temperature than model R3 in order to prevent the cloud from gravitational collapse. The core temperature is about $T=230 \mathrm{~K}$, rises up to $T=920 \mathrm{~K}$ at $r=$ $10 \mathrm{pc}$ and reaches $T=1000 \mathrm{~K}$ at $r=30 \mathrm{pc}$. Heat conduction is very sensitive to a rise in temperature $\left(\kappa \propto T^{5 / 2}\right.$, see Eq. (2)) so that energy transport into the core region is expected.

The evolution of the density distribution is shown in Fig. 14. A transition region at the cloud edge has formed with an extension almost constant after a formation time of about $15 \mathrm{Myr}$. The structure of this layer is much more regular than in the previous models without self-gravity where RT instabilities dominate the region. Self-gravity is a process that stabilizes the cloud against RT instability (Murray et al. 1993): the effective acceleration which the surface material experiences as the net effect of evaporation (like in models R2 and R3) vs. self-gravity points radially inwards as the density gradient does.

Similar to model R3 the cloud can be devided into an inert core, a heat-conduction dominated zone with nearly homogeneous density distribution and the transition zone. They are visible by the density profile after $53 \mathrm{Myr}$ in comparison to the initial model (Fig. 15) and the corresponding temperature profiles (Fig. 16).

Because of gravitation the density profile of the intermediate zone is not as flat as the temperature profile but approaches hydrostatic equilibrium.

During the whole simulation energy is transported into the cloud at $r>18$ pc due to heat conduction. This energy flow is connected with a mass transport because of the induced overpressure. This mass flow is neither locally nor temporally constant but corresponds to the temperature of the transition zone. 


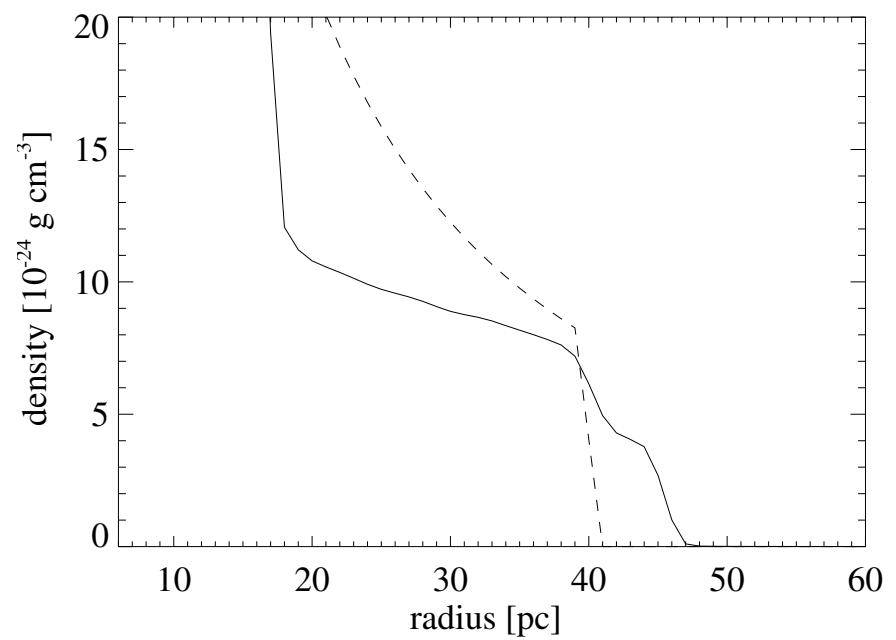

Fig. 15. Density profile of the model R4 at a time $t=53 \mathrm{Myr}$ (solid curve) in comparison with the initial model (dashed).

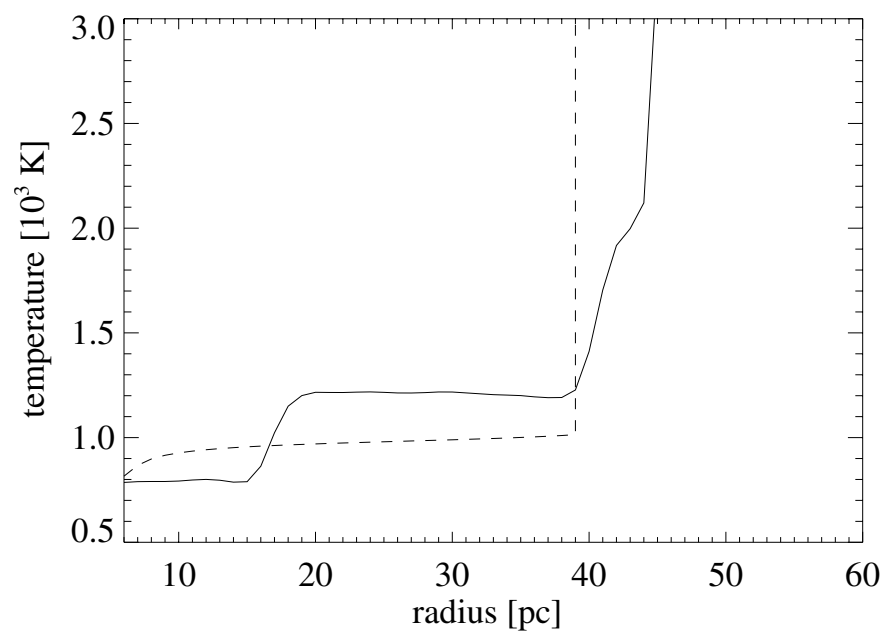

Fig. 16. Temperature profile of the model $\mathrm{R} 4$ at a time $t=53 \mathrm{Myr}$ (solid curve) in comparison with the initial model (dashed).

To visualize this correlation, the mass flow and the temperature at $r=40 \mathrm{pc}$ (inner boundary of the transition zone) are plotted in Fig. 17.

Similar to the previous models the evaporation of cloud material leads to a repulsion and therefore triggers a pressure wave inside the cloud. The consequence of the inward-travelling wave, namely, the negative $\dot{m}$ is visible in Fig. 17 (e.g.: $t=27$ Myr) and correlated with a rise in temperature of the transition zone. The wave interacts with the density discontinuity at $r=18 \mathrm{pc}$ (e.g.: $t=30 \mathrm{Myr}$ ), is reflected, travels outwards, reenters the transition zone and, with that, pushes cold cloud material into the transition zone (e.g.: $t=32.5 \mathrm{Myr}$ ). While material continuously flows into the transition layer the temperature there is further reduced (e.g.: $32.5<t<42 \mathrm{Myr}$ ). This transport is stopped as soon as a new hydrostatic equilibrium has been adjusted. The temperature fluctuations at the inner boundary of the transition zone also become visible at the temperature distribution outside the cloud by means of the evaporated mass flow. E.g. at $r=95 \mathrm{pc}$ the temperature varies by $2 \%$ correlated with the evaporation rate of about $1.5 \times 10^{-4} M_{\odot} \mathrm{yr}^{-1}$ on average (see Fig. 18). This is slightly larger than in model R3 because the area feeding the evaporation is larger than in the previous model.

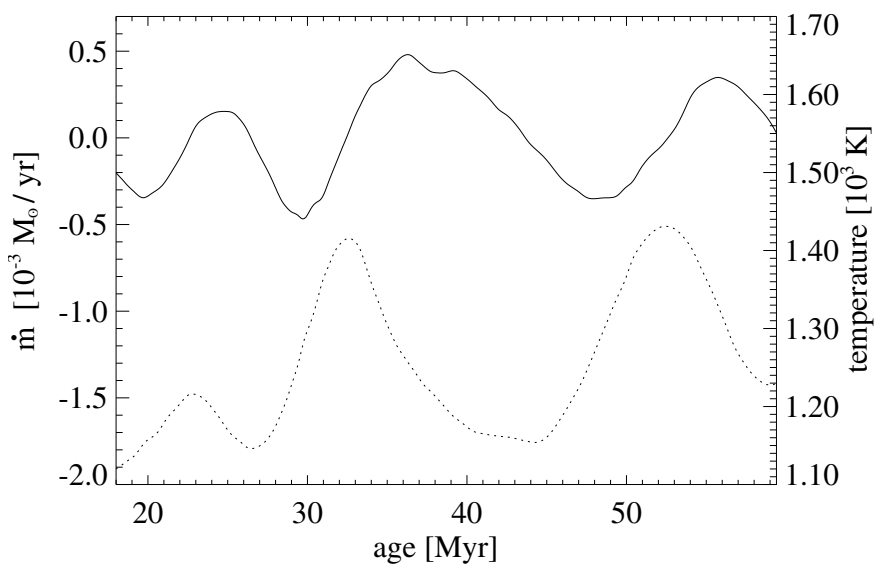

Fig. 17. Evolution of the mass-loss rate (solid curve) and the temperature (dotted) at the inner boundary of the transition zone $(r=40 \mathrm{pc})$ for model R4.

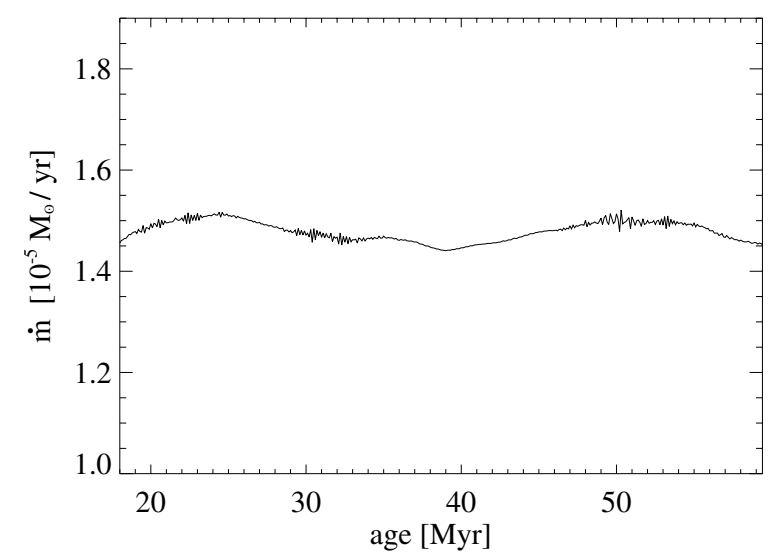

Fig. 18. Evaportion rate for the model R4.

This study shows that dynamical processes inside the cloud must not be neglegted but determine the transport inside the transition zone and with this the temperature profile at the cloud edge. Even far away from the cloud this dynamics can be comprehended by the mass-loss rate or temperature distribution. Because of the much larger sound velocity than that of the evaporation flow the external temperature fluctuation affect the evaporaton rate.

\subsection{Model R5}

Because already the studies by McKee \& Cowie (1977) indicate that a reduction of the heat flux due to cooling processes is expected but only for clouds with $\sigma_{0}<0.027$, model R5 takes heating and cooling processes into account. Opposite to CM77 this model is calculated with self-gravity. From model R4 we conclude that self-gravity acts only as a smoothing agent for reducing instabilities that occur when heated material is evaporated from the cloud surface into the ICM. The massloss rate was not significantly altered by this process. So, this model should be comparable to the analytic model of Cowie and McKee. In models R2-R4 heat conduction leads to a temperature and therefore to a pressure increase at the edge of the cloud where the transition zone forms between the cold cloud and the hot ICM. In order to compensate the local energy input, two processes are physically plausible: the cloud distributes the energy throughout its volume by dynamical transport processes and/or it emits the energy by the process of radiative cooling. Cooling 


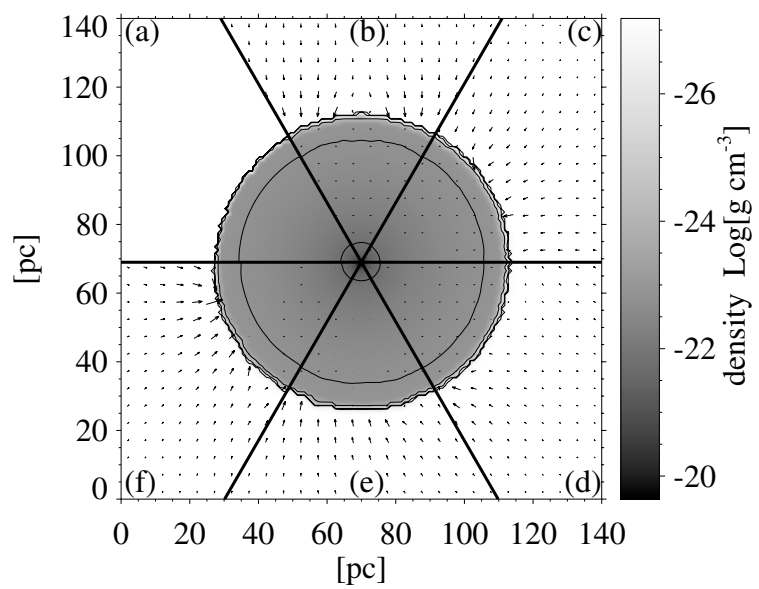

Fig. 19. Evolution of the density distribution of model R5 after 0 Myr a), $5 \mathrm{Myr}$ b), $10 \mathrm{Myr}$ c), $15 \mathrm{Myr}$ d), $20 \mathrm{Myr}$ e) and $25 \mathrm{Myr}$ f). Lines of equal density are drawn between $\rho=10^{-22}$ and $10^{-26} \mathrm{~g} \mathrm{~cm}^{-3}$ in steps on 1 dex.

is very sensitive to the particle density and is most effective in regions with high density. The question is therefore whether the cooling efficiency is large enough to alter the formation or evolution of the transition zone and, with that, to change the evaporation rate.

There are two main differences visible in the evolution of the density distribution of model R5 (Fig. 19) in comparison to the previous models. First of all, the velocity field outside the cloud is directed towards the cloud that clearly indicates accretion of ICM onto the cloud's surface. Secondly, the density distribution inside the cloud remains nearly unaffected by heat conduction. Similar to the models R2-R4 a transition zone is formed but its extension is much narrower. This means that the influence of heat conduction is restricted to only a thin layer around the cloud with a thickness of only few parsecs.

The used cooling function shows a steep increase for $T$ around $10^{4} \mathrm{~K}$. For regions with temperatures below $10^{4} \mathrm{~K}$ this means that they can be heated up to about $10^{4} \mathrm{~K}$ with high efficiency but then should somewhere easily find an energetic balance between heating and cooling. This equilibrium situation is determined by the Field length $\lambda_{\mathrm{F}}$ (Eq. (8)). In the outermost shell of the cloud $\lambda_{\mathrm{F}}$ amounts to $1.4 \times 10^{3} R_{\text {cld }}$ (Vieser $\&$ Hensler 2005; hereafter: Paper II). For this reason the occurence of RT instability is prevented and no further refined grid resolution is neccesary. Due to the density gradient towards the cloud core $\lambda_{\mathrm{F}}$ drops within an interface formed by heat conduction down to values much smaller than $R_{\text {cld }}$. Because of its high cooling efficiency by larger density a temperature of $8000-10^{4} \mathrm{~K}$ is achieved at which its thermal energy density is not exceeding the gravitational one. As a consequence, the interface does not dissolve from the cloud but accumulates surrounding gas that enters the interface by means of heat conduction.

From Fig. 20 one can recognize that between $30 \mathrm{Myr}$ and $40 \mathrm{Myr}$ a quasi-stationary temperature distribution has been adjusted. The balance at which the energy input due to heat conduction plus heating is compensated by cooling is reached at temperatures of $10^{4} \mathrm{~K}$ and radii $r_{\star}$. For the reached values at $r_{\star}$ the Field length amounts to not more than $10^{-2} R_{\text {cld }}$. This effect has two consequences: at first, the physical one is that the parameter regime of condensation is reached; secondly, the cell size is larger than the typical thermodynamical length scale, i.e. here the mean free path of electrons and ions, respectively.

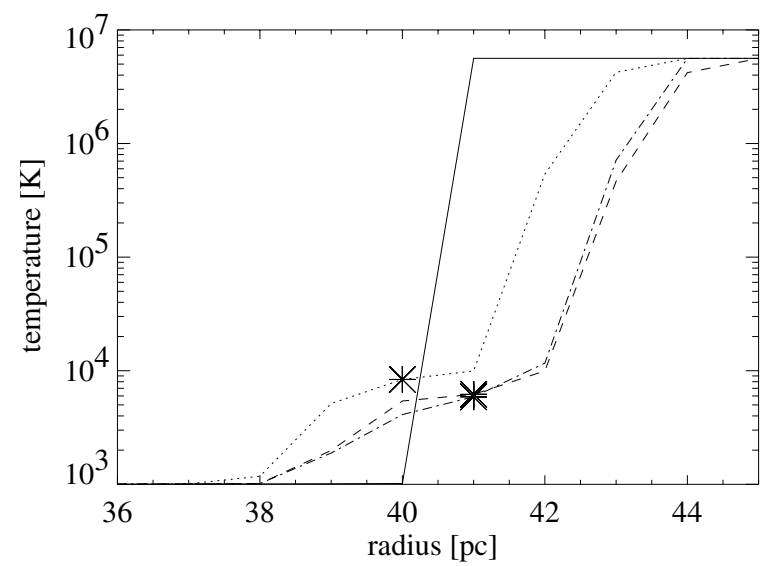

Fig. 20. Comparison of temperature profiles of model R5 at different ages: initial model (solid curve), after $30 \mathrm{Myr}$ (dotted), after $40 \mathrm{Myr}$ (dashed) and after $55 \mathrm{Myr}$ (dashed-dotted). The radii $\left(r_{\star}\right)$ at which the energy input due to heat conduction plus radiative heating is balanced by radiative cooling are marked by crosses.

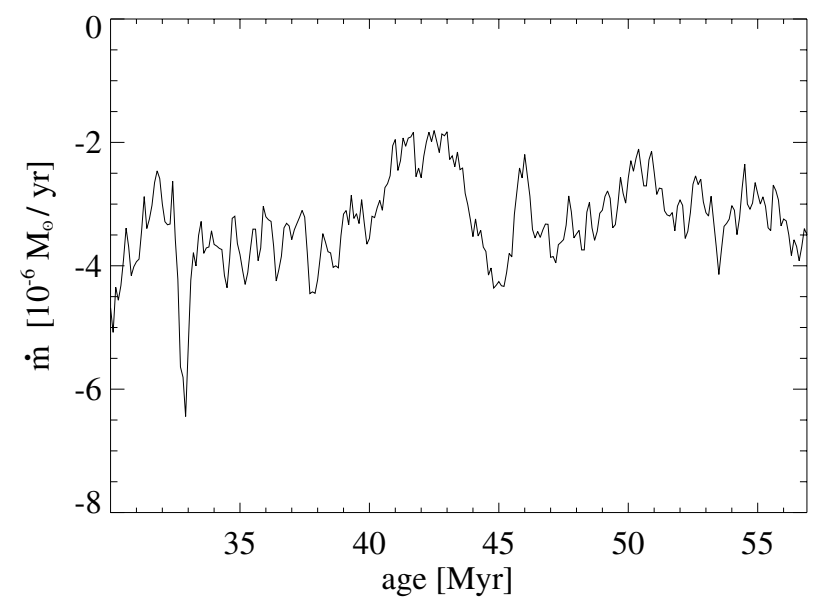

Fig. 21. Evolution of the mass-loss rate of model R5.

Therefore, this means that the hydrodynamical treatment is appropriate (Koyama \& Inutsuka 2004).

Thermal energy is transported from $r>r_{\star}$ inwards during the whole calculation and finds an equilibrium with the cooling rate at $r_{\star} \approx 41 \mathrm{pc}$ and $8000 \mathrm{~K}$. Consequently, condensation occurs (Fig. 21) in this model with a value of about $-3.5 \times 10^{-6} M_{\odot} \mathrm{yr}^{-1}$. Although the available conductive energy at $r<r_{\star}$ is reduced the temperature rises moderately above $10^{3} \mathrm{~K}$ for $r>38 \mathrm{pc}$ (Fig. 20), by this generating a turbulent velocity field that transports the accreted material from the cloud surface towards deeper layers. Due to the condensation of ICM onto the cloud and its transport inside the cloud, the mass fraction of ICM is rising continuously. The distribution of ICM in comparison to the whole cloud mass is visualized in Fig. 22. A growing shell becomes visible with an ISM mass fraction of at least $1 \%$. After $50 \mathrm{Myr}$ this layer has reached a thickness of about $10 \mathrm{pc}$.

To emphasise, heat conduction together with cooling processes does not only change the mass flow from evaporation to condensation but also provide a new possibility to enrich the cloud with ICM and to distribute it throughout large parts of the cloud. 


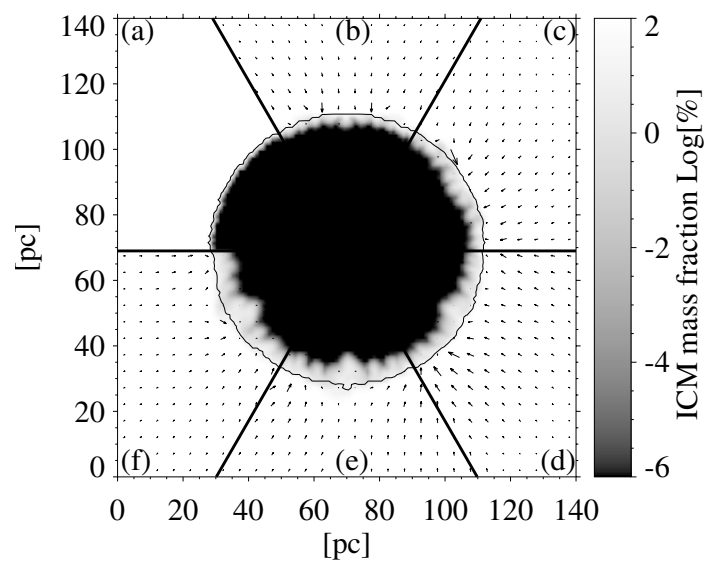

Fig. 22. Evolution of the ICM mass fraction $\left(\rho_{\text {ISM }} / \rho_{\text {cld }}\right)$ of model R5 after $0 \mathrm{Myr}$ a), $10 \mathrm{Myr}$ b), $20 \mathrm{Myr}$ c), $30 \mathrm{Myr}$ d), $40 \mathrm{Myr}$ e) and $50 \mathrm{Myr}$ f). The solid line represents the cloud boundary.

Table 2. Mass-loss rates of the various simulations (negative values mean mass accretion!).

\begin{tabular}{cc}
\hline \hline Model & $\dot{m}\left[M_{\odot} \mathrm{yr}^{-1}\right]$ \\
\hline R1 & $0.8 \times 10^{-3}$ \\
R2 & $1.4 \times 10^{-5}$ \\
R3 & $1.2 \times 10^{-5}$ \\
R4 & $1.4 \times 10^{-5}$ \\
R5 & $-3.5 \times 10^{-6}$ \\
\hline
\end{tabular}

\section{Discussion}

In this paper we investigate the evolution of interstellar clouds that are resting in a hot rarefied medium. Models with different density structures and various physical processes are compared with the aid of numerical simulations. The classical evaporation rate without taking saturation effects into account amounts to $10^{-3} M_{\odot} \mathrm{yr}^{-1}$. Analytical consideration of saturation allows to lower it to about $5.6 \times 10^{-4} M_{\odot} \mathrm{yr}^{-1}$ (CM77) or $3.7 \times 10^{-4} M_{\odot} \mathrm{yr}^{-1}$ (DB93), respectively.

The global saturation parameter of heat conduction in all calculations here was $\sigma_{0}=4.88$. The mass-loss rates deduced from the simulations are summarized in Table 2. In order to compare with analytical studies (CM77, DB93), the initial model R1 shows a homogeneous density distribution and a temperature determined by pressure equilibrium. Influences by self-gravity or heating and cooling are neglected. As a first step, in model R1 only the classical description of heat conduction is taken into account. The mass-loss rate is in good agreement with the analytical results, but we learn that it oscillates due to excited cloud pulsations.

The evolution of a cloud taking saturation effects into account (model R2) proceeds not as violent as in R1. The energy input into the cloud is reduced due to the saturated heat flux. Nevertheless the cloud's outermost layer is heated up and forms a transition layer between the cold cloud and the hot ICM in which the material can remain near pressure equilibrium with the ICM. From there cloud gas is dissolved and pushed into the hot ICM at subsonic speed. This, however, changes the temperature and density profiles in the cloud's environment drastically in comparison to the initial model, so that it is not surprising that the mass-loss rate is reduced to about one order of magnitude below the analytical value (CM77, DB93). On the other hand, this value is similar to the one derived analytically for $\sigma_{0} \approx 100$ and indeed the mean local saturation parameter deduced from the simulated temperature profile yields about 100 in contrast to 4.88 set as the formal value. From that we learn, that the global saturation parameter is therefore not conclusive to derive the mass-loss rate.

The influence of a multi-phase density structure of the cloud on the evaporation rate is not significant (model R3). Since density and temperature at the cloud surface are similar to the previous model which serve as the dominant factors for the heat input and by this for the mass loss rate, the same result is expected.

That RT instability is the responsible mechanism that shapes the clouds' surfaces in models R2 and R3 becomes plausible, because in model R4 the acceleration by self-gravity overcomes the outwards directed acceleration of the expanding shell. Thus, the shape of the cloud remains undisturbed but the mass-loss rate is slightly higher than in model R3 because the temperature near the cloud core of the initial model is higher for hydrostatic equilibrium. By this, heat conduction can affect deeper layers of the cloud.

In model R5 heating and cooling processes are added. Since the used cooling function increases steeply at $10^{4} \mathrm{~K}$ the heating due to heat conduction can be balanced by radiative cooling at around $10^{4} \mathrm{~K}$ for sufficiently high densities. In the presented cloud model saturated heat conduction is not able to transport enough thermal energy into the cloud in order to rise the temperture above $10^{4} \mathrm{~K}$ and therefore to evaporate the cloud. The cloud rim remains at temperatures of about $10^{4} \mathrm{~K}$. Correlated with the energy transport onto the cloud's surface, mass is accumulated from the ISM and condenses onto the cloud. The cloud regions near the surface are slightly heated so that a turbulent velocity field emerges that mixes the accreted ICM into deeper layers.

Three major conclusions can be drawn from the numerical models presented in this paper: 1) Evaporation itself alters the environmental conditions in the sense of positive feedback. 2) The assumption of classical heat conduction is invalid under real conditions of interstellar clouds. In contradiction to those analytical results, heat conduction is reduced to the saturated limit. 3) In connection with radiative cooling this can lead to condensation of hot ICM onto clouds in a regime of cloud parameters where evaporation is requested from the analytical approach. By this it provides an alternative way to accrete and mix ICM with cloudy material.

Acknowledgements. The authors thank Tim Freyer for stimulating discussions and Tomek Plewa for providing us with his numerical code solving the heat conduction equation. We gratefully acknowledge suggestions by an anonymous referee which helps for clarification of the results.

This work was partly supported by the Deutsche Forschungsgemeinschaft (DFG) under grant numbers He 1487/5-3 and He 1487/25-1. The computations were performed at the Rechenzentrum der Universität Kiel, the Konrad-ZuseZentrum für Informationstechnik in Berlin, and the John von Neumann-Institut für Computing in Jülich.

\section{References}

Begelman, M. C., \& McKee, C. F. 1990, ApJ, 358, 375

Black, J. H. 1987, in Interstellar Processes, ed. D. J. Hollenbach, \& H. A. Thronson (Kluwer: Dordrecht), 731

Böhringer, H., \& Hensler, G. 1989, A\&A, 215, 147

Brüns, C., Kerp, J., Kalberla, P. M. W., \& Mebold, U. 2000, A\&A, 357, 120

Brüns, C., Kerp, J., \& Pagels, A. 2001, A\&A, 370, L26

Campbell, P. M. 1984, Phys. Rev. A, 30, 365

Chevalier, R. A. 1975, ApJ, 200, 698

Cowie, L. L., \& McKee, C. F. 1977, ApJ, 211, 135 (CM77)

Crank, J., \& Nicolson, P. 1947, Proc. Cambridge Phil. Soc., 43, 50

Dalgarno, A., \& McCray, R. A., 1972, ARA\&A, 10, 375

Dalton, W. W., \& Balbus, S. A. 1993, ApJ, 404, 625 (DB93)

Danly, L., Albert, C. E., \& Kuntz, K. D. 1993, ApJ, 416, L29 
de Jong, T. 1977, A\&A, 55, 137

de Jong, T., Dalgarno, A., \& Boland, W. 1980, A\&A, 91, 68

Field, G. B., Goldsmith, D. W., \& Habing, H. J. 1969, ApJ, 155, L149

Graham, R., \& Langer, W. D. 1973, ApJ, 179, 469

Heyer, M. H., Brunt, C., Snell, R., et al. 1996, ApJ, 464, L175

Juncosa, M. L., \& Young, D. 1957, Proc. Cambridge Phil. Soc., 53, 448

Khan, S. S., \& Rognlien, T. D. 1981, Phys. Fluids, 24, 1442

Keenan, F. P., Shaw, C. R., Bates, B., et al. 1995, MNRAS, 272, 599

Koyama, H., \& Matsuka, S.-I. 2004, ApJ, 602, L25

Manheimer, W. M., \& Klein, H. H. 1975, Phys. Fluids, 18, 1299

Matte, J. P., \& Virmont, J. 1982, Phys. Rev. Lett., 49, 1936

McKee, C. F., \& Cowie, L. L. 1977, ApJ, 215, 213

McKee, C. F., \& Ostriker, J. P. 1977, ApJ, 218, 148

Morse, R. L., \& Nielsen, C. W. 1973, Phys. Fluids, 16, 909

Murray, S. D., White, S. D. M., Blondin, J. M., \& Lin, D. N. C. 1993, ApJ, 407, 588

Normandeau, M., Taylor, A. R., \& Dewdney, P. E. 1996, Nature, 380, 687

Penston, M. V., \& Brown, F. E. 1970, MNRAS, 150, 373

Pietz, J., Kerp, J., Kalberla, P. M. W., et al. 1996, A\&A, 308, L37

Press, W. H., Teukolsky, S. A., Vetterling, W. T., \& Flannery, B. P. 1986, Numerical Recipes (New York: Cambridge University Press)
Raymond, J. C., Cox, D. P., \& Smith, B. W. 1976, ApJ, 204, 290

Rozyczka, M. 1985, A\&A, 143, 59

Samland, M., Hensler, G., \& Theis, C. 1997, ApJ, 476, 544

Slavin, J. D., \& Cox, D. P. 1992, ApJ, 392, 131

Spitzer, L. 1962, Physics of Fully Ionized Gases, Interscience, New York

Taylor, A. R., Irwin, J. A., Matthews, H. E., \& Heyer, M. H. 1999, ApJ, 513, 339

van Leer, B. 1977, J. Comp. Phys., 23, 276

van Woerden, H., Wakker, B. P., Schwarz, U. J., et al. 1997, in Proc. of the IAU

Coll. No. 166, The Local Bubble and Beyond, ed. D. Breitschwerdt, M. J.

Freyberg \& J. Trümper (New York: Springer), 467

Vieser, W., \& Hensler, G. 2007, A\&A, 472, 141 (Paper II)

Wakker, B. P., \& Schwarz, U. J. 1991, A\&A, 250, 484

Wakker, B. P., \& van Woerden, H. 1997, ARA\&A, 35, 217

Yanenko, N. N. 1971, The method of fractional steps, ed. M. Holt (New York: Springer)

Yorke, H. W., \& Welz, A. 1996, A\&A, 315, 555

Zel'dovich, Y. B., \& Pikel'ner, S. B. 1969, Sov. Phys.-JETP, 29, 170

Zel'dovich, Y. B., Raizer, Y. P., Hayes, W. D., \& Probstein, R. F. 1967, Physics of Shock Waves and High-Temperature Hydrodynamic Phenomena, (New York: Academic Press) 
W. Vieser and G. Hensler: Evaporation and condensation of spherical interstellar clouds, Online Material $p 1$

\section{Online Material}


W. Vieser and G. Hensler: Evaporation and condensation of spherical interstellar clouds, Online Material p 2

\section{Appendix A: Heat conduction - implementation and tests}

The implementation of heat conduction into the existing and tested hydro-code turned out to be more complicated than expected in order not to limit the hydro-timestep $\tau_{\text {hyd }}$ to the conduction-timestep given by

$\tau_{\text {cond }}=\frac{e}{T \kappa}[\min (\mathrm{d} r, \mathrm{~d} z)]^{2}$

where $\mathrm{d} r$ and $\mathrm{d} z$ are the distances between the centers of two neighbouring cells in $r$ and $z$ direction respectively. The problem dealing with these incompatible timescales is treated by using a semi-implicit scheme to solve the heat conduction equation.

The equation describing the change of the internal energy density due to heat conduction

$$
\frac{\partial e}{\partial t}=\frac{1}{r} \frac{\partial}{\partial r}\left[r \kappa \frac{\partial T}{\partial r}\right]+\frac{\partial}{\partial z}\left[\kappa \frac{\partial T}{\partial z}\right]
$$

is transformed via the equation of state for an ideal gas into an expression for the change of the temperature:

$\frac{\partial T}{\partial t}=\frac{T}{e} \frac{1}{r} \frac{\partial}{\partial r}\left[r \kappa \frac{\partial T}{\partial r}\right]+\frac{T}{e} \frac{\partial}{\partial z}\left[\kappa \frac{\partial T}{\partial z}\right]$.

Both spatial directions are treated seperately using the same strategy. Therefore only the solution of the radial part is described in the following: The new temperature distribution is calculated for all cells in the $r$-direction with a fixed $z$-coordinate. This is done using an ADI-method. After the temperature in this row has converged, the index of the $z$-coordinate is increased by one and the equation is solved for the next row.

If the discretisation of Eq. (A.3) is done in a pure explicit way not only the timestep is limited to the conductiontimestep. This procedure by itself is numerically unstable (Crank \& Nicolson 1947). Instead of this approach we used a semi-implicit procedure where the implicit part is weighted by a factor $0.5<\alpha<1$ and the explicit part by a factor $1-\alpha$ what results in:

$$
\begin{aligned}
\frac{T_{i, j}^{n+1}-T_{i, j}^{n}}{\Delta t}= & (1-\alpha)\left\{\frac{2}{r_{i, j}+r_{i, j+1}} \frac{T_{i, j}^{n}}{e_{i, j}^{n}} \frac{1}{\Delta r}\right. \\
& \times\left[\left(r_{i, j+1} \kappa_{i, j+1}^{n} \frac{T_{i, j+1}^{n}-T_{i, j}^{n}}{\Delta r}\right)\right. \\
& \left.\left.-\left(r_{i, j} \kappa_{i, j}^{n} \frac{T_{i, j}^{n}-T_{i, j-1}^{n}}{\Delta r}\right)\right]\right\} \\
& +\alpha\left\{\frac{2}{r_{i, j}+r_{i, j+1}} \frac{T_{i, j}^{n+1}}{e_{i, j}^{n+1}} \frac{1}{\Delta r}\right. \\
& \times\left[\left(r_{i, j+1} \kappa_{i, j+1}^{n+1} \frac{T_{i, j+1}^{n+1}-T_{i, j}^{n+1}}{\Delta r}\right)\right. \\
& \left.\left.-\left(r_{i, j} \kappa_{i, j}^{n+1} \frac{T_{i, j}^{n+1}-T_{i, j-1}^{n+1}}{\Delta r}\right)\right]\right\} .
\end{aligned}
$$

This equation can be rearranged and the different coefficients be combined to form:

$A_{i, j} T_{i, j-1}^{n+1}+B_{i, j} T_{i, j}^{n+1}+C_{i, j} T_{i, j+1}^{n+1}=D_{i, j}$.

For a row with the index $i$ kept constant this describes a maxtrix equation which can be solved with standard methods (e.g.

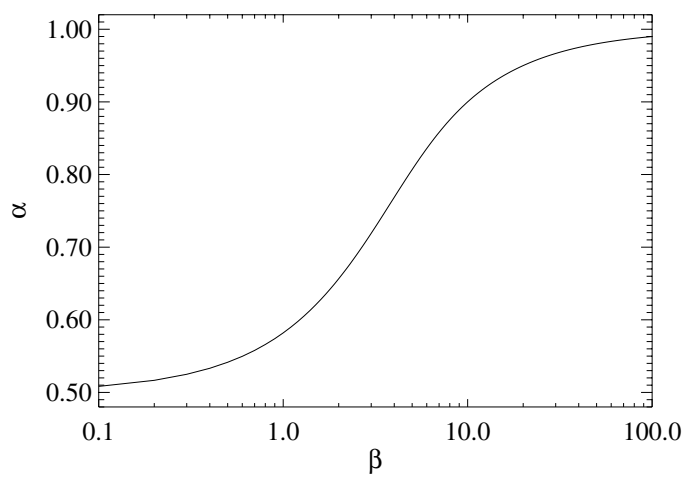

Fig. A.1. Weightning factor $\alpha$ as a function of $\beta$.

Press et al. 1986). We used an ADI-method. The weightning parameter $\alpha$ is chosen in a manner so that the implicit part is pronounced if the conduction-timestep is much smaller than the hydro-timestep. This means that high temperatures or large conductivities dominate the integration domain. The ratio between the two timesteps is defined as $\beta$

$\beta=\frac{\tau_{\text {hyd }}}{\tau_{\text {cond }}}$,

that deals as a quantity to derive $\alpha$ :

$\alpha=\frac{1-\beta-\exp [-\beta]}{\beta(\exp [-\beta]-1)}$,

with the additional limitation

$\alpha=\left\{\begin{array}{rcc}0.5 & : & \alpha<0.5 \\ \alpha & : & 0.5 \leq \alpha \leq 1 \\ 1 & : & \alpha>1\end{array}\right.$

so that the minimal implicit portion is $50 \%$. The course of $\alpha$ as a function of $\beta$ is plotted in Fig. A.1.

The temperature distribution calculated is this way results from the assumption that the conductivities remains constant during the iteration of Eq. (A.4). This is not the case as $\kappa$ is strongly temperature dependent. Therefore, after the new temperature distribution is calculated, the conduction coefficients have to be updated for this new temperatures and Eq. (A.4) has to be solved again. This procedure is repeated until the error between the new temperatures and the old ones is less then $10^{-4}$.

We have tested the method described above through the computation of problems with known solutions. Two test cases were run, one with a constant $\kappa$ and one with $\kappa \propto T^{m}$ with $m=5 / 2$ for which a self similar solution exists (Zel'dovich et al. 1969). The quality of the results for both test cases are similar so only the results of the $\kappa=$ const.-case are presented here.

In this test the plasma is assumed permanently static and with a uniform and "frozen" density distribution with a particle density $n=6.6 \times 10^{-4} \mathrm{~cm}^{-3}$. We have considered the propagation of a spherical pure conduction front in a uniform medium. For this problem an analytic solution for the temperature distribution as function of time is available:

$T(r, t)=\left(\frac{Q}{4 \pi \kappa t}\right)^{1.5} \exp \left[\frac{-r^{2}}{4 Q t}\right]$.

We have calculated the numerical solution in a cylindrical coordinate system, taking as initial profile the analytical solution at $t=0.5 \mathrm{~s}$, for $Q=10^{54} \mathrm{~K} \mathrm{~cm}^{-3}$ and $\kappa=1.36 \times 10^{12}$ what corresponds to a conductivity of a plasma at a temperature of 
W. Vieser and G. Hensler: Evaporation and condensation of spherical interstellar clouds, Online Material p 3

$5.6 \times 10^{6} \mathrm{~K}$. The grid is $200 \times 100$ cells with $-0.032<z<$ $0.032 \mathrm{pc}$ and $0<r<0.032 \mathrm{pc}$. The resulting propagation, compared with the analytical solution, and the relative error is shown in Fig. A.2 for different times. The relative error within the hottest region of the plasma amounts to about 2-3\%. Only at the edge of the conduction front where the temperature is some orders of magnitudes lower than in the inner regions the error is slightly increased. In Figs. A.2d-f the influence of the grid boundary is visible. Here the temperature gradient is forced to be zero. With these studies we could verify the validity and accuracy of the code. 
W. Vieser and G. Hensler: Evaporation and condensation of spherical interstellar clouds, Online Material p 4
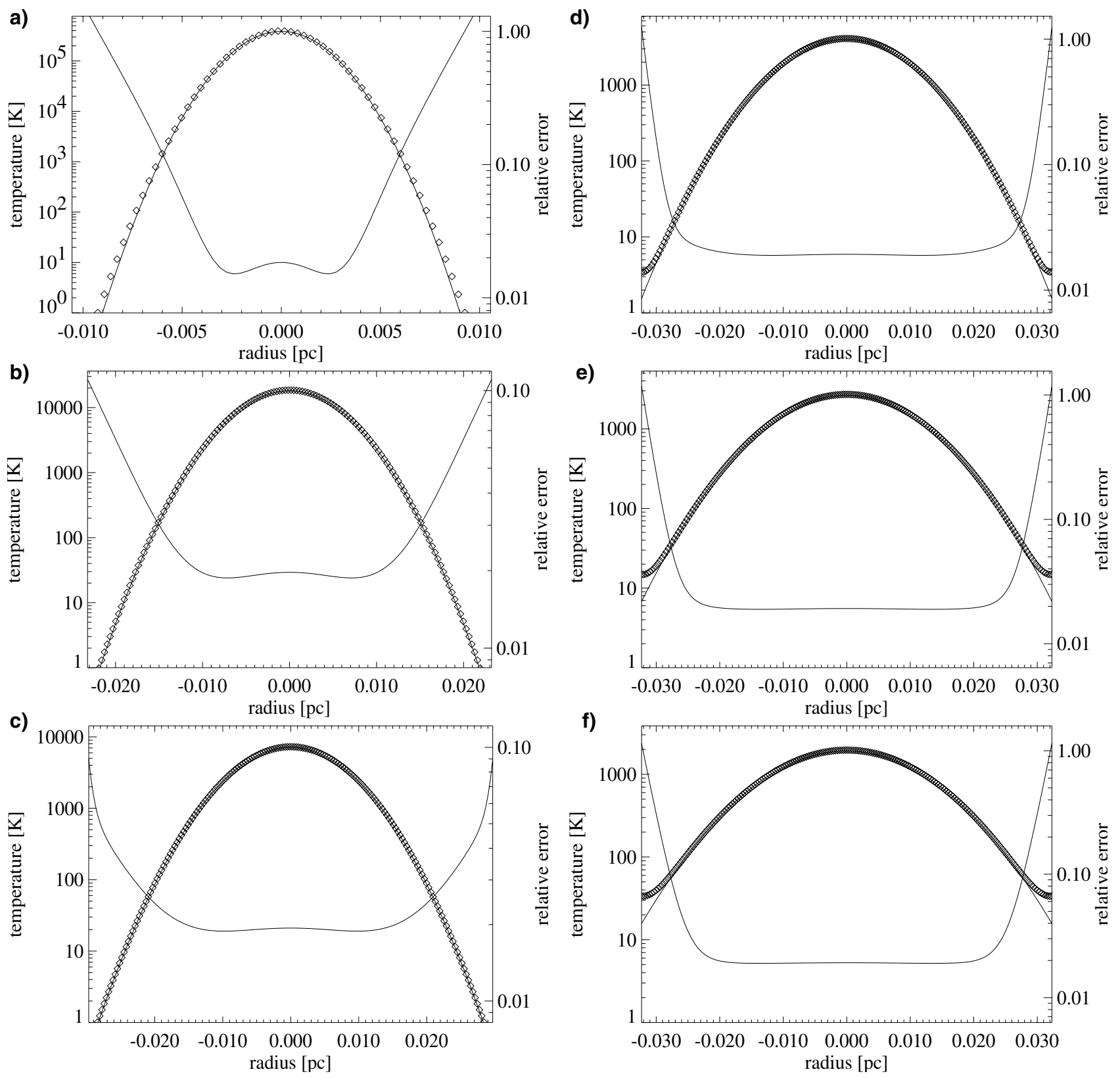

Fig. A.2. Propagation of the conduction front after $1.0 \mathrm{~s}$ a), $6.0 \mathrm{~s} \mathrm{~b}$ ), $11 \mathrm{~s} \mathrm{c}$ ), $16 \mathrm{~s} \mathrm{~d}$ ), $21 \mathrm{~s}$ e) and $26 \mathrm{~s}$ f). Comparison of the numerical results (diamonds) with the analytical solution (solid line). Additionally, the relative error between both results is plotted (convex line). 